\title{
A survey for pulsating subdwarf B stars with the Nordic Optical Telescope ${ }^{\star}$
}

\author{
R. H. Østensen ${ }^{1}$, R. Oreiro ${ }^{1}$, J.-E. Solheim ${ }^{2}$, U. Heber ${ }^{3}$, R. Silvotti ${ }^{4}$, J. M. González-Pérez ${ }^{5}$, \\ A. Ulla ${ }^{6}$, F. Pérez Hernández ${ }^{5,7}$, C. Rodríguez-López ${ }^{6,8}$, and J. H. Telting ${ }^{9}$
}

1 Instituut voor Sterrenkunde, K. U. Leuven, Celestijnenlaan 200D, 3001 Leuven, Belgium e-mail: [roy; raquel]@ster. kuleuven.be

2 Institutt for Teoretisk Astrofysikk, Universitetet i Oslo, 0212 Blindern-Oslo, Norway

3 Dr. Remeis-Sternwarte, Astronomisches Institut der Univ. Erlangen-Nürnberg, 96049 Bamberg, Germany

4 INAF-Osservatorio Astronomico di Torino, Strada dell'Osservatorio 20, 10025 Pino Torinese, Italy

5 Instituto de Astrofísica de Canarias, 38200 La Laguna, Tenerife, Spain

6 Departemento Física Aplicada, Universidade de Vigo, 36310 Vigo, Spain

7 Departamento de Astrofísica, Universidad de La Laguna, 38205 La Laguna, Tenerife, Spain

${ }^{8}$ Laboratoire d'Astrophysique de Toulouse-Tarbes, Univ. de Toulouse, 14 Av. Edouard Belin, 31400 Toulouse, France

9 Nordic Optical Telescope, 38700 Santa Cruz de La Palma, Spain

Received 15 October 2009 / Accepted 6 January 2010

\section{ABSTRACT}

\begin{abstract}
Context. A search programme for pulsating subdwarf B stars was conducted with the Nordic Optical Telescope on La Palma over 59 nights between 1999 and 2009.

Aims. The purpose of the programme was to significantly extend the number of rapidly pulsating sdB stars to better understand the properties of this new group of variable compact stars.

Methods. Candidates were selected initially from the HS and HE surveys, but were supplemented with additional objects from other surveys. Short sequences of time-series photometry were made on the candidates to determine the presence of rapid pulsations.

Results. In total twenty new pulsators were found in this survey, most of which have already been published and some extensively studied. We present four new short period pulsators, bringing the total of such pulsators up to 49. We also give limits on pulsation amplitudes for 285 objects with no obvious periodic variations, summarise the results of the survey, and provide improved physical parameters on the composite pulsators for which only preliminary estimates were published earlier.
\end{abstract}

Key words. subdwarfs - surveys - stars: oscillations

\section{Introduction}

Hot subluminous stars are considered to be extreme horizontal branch (EHB) stars or closely related objects, with effective temperatures $T_{\text {eff }} \simeq 20-35 \mathrm{kK}$. The EHB models imply that they are core helium burning objects with an extremely thin $\left(M_{\mathrm{env}} \leq 0.02 M_{\odot}\right)$ inert hydrogen dominated envelope (Heber 1986; Saffer et al. 1994). This structure prevents them from ascending the asymptotic giant branch (AGB), and they must evolve instead towards higher temperatures and surface gravities after their core helium is exhausted. Thus, an sdB star crosses the hotter sdO domain before reaching degeneracy and cooling as a normal white dwarf star (Dorman et al. 1993). However, important questions remain concerning the exact evolutionary paths and the appropriate timescales.

How they evolve to the EHB configuration is controversial. The problem is how the mass loss mechanism in the progenitor manages to remove all but a tiny fraction of the hydrogen envelope at about the same time as the core has attained the mass $\left(\sim 0.47 M_{\odot}\right)$ required for the He flash. About half of the sdB stars reside in close binary systems, with either a white dwarf or an M-dwarf as companion (Maxted et al. 2001;

* Full Table 5 is only available in electronic form at the CDS via anonymous ftp to

cdsarc.u-strasbg.fr (130.79.128.5) or via

http://cdsweb.u-strasbg.fr/cgi-bin/qcat?J/A+A/513/A6
Napiwotzki et al. 2004), and a significant fraction of the rest are in wider binaries with a main sequence $\mathrm{F}-\mathrm{K}$ star as companion. Therefore mass transfer in close binary evolution must be an important evolutionary pathway leading to the formation of $\mathrm{sdB}$ stars (Han et al. 2002, 2003). A recent review of hot subdwarf stars is provided by Heber (2009).

The discovery of multimode pulsations in sdBs opened an attractive opportunity of probing their interiors with seismological methods. The properties of the sdB pulsators (sd$\mathrm{BV}$ s or $\mathrm{V} 361 \mathrm{Hya}^{1}$ stars after the prototype; Kilkenny et al. 1997) are characterised by relatively short pulsation periods ( $\sim 1$ to $10 \mathrm{~min}$ ) and low pulsation amplitudes attributed to low order pressure $(p)$ modes. Most V361 Hya stars have been found with pulsation amplitudes of around ten millimodulation amplitudes $\left(\mathrm{mma}^{2}\right)$, although a few objects show a main peak amplitude up to $60 \mathrm{mma}$ (V338 Ser, Kilkenny et al. 1999; Balloon 090100001, Oreiro et al. 2004), while the lowest level pulsator discovered up to now is LM Dra (Silvotti et al. 2000), with no peaks above $2 \mathrm{mma}$. The number of detected periods varies from a single one to more than fifty, strongly correlated with the accuracy of the measurements.

\footnotetext{
1 Translations between the old survey names and the variable star names used here are provided in Table 9.

2 The mma units are $10^{-3}$ of the Fourier amplitudes of a light-curve in normalised intensity units. One mma translates to a peak-to-peak amplitude of two millimodulation intensity (mmi) units in the light curve.
} 
In 2001, some sdBs were discovered to show long-period ( $\sim 1 \mathrm{~h}$ ) photometric modulations (Green et al. 2003), which were interpreted as high radial order gravity $(g)$ modes. These stars are referred to as long period sdBV stars or V1093 Her stars after the prototype. V1093 Her stars are cooler than their short-period counterparts, although their instability regions appear to overlap around $T_{\text {eff }} \sim 29 \mathrm{kK}$, where they can display simultaneous short and long period pulsations. These stars are referred to as hybrid sdBVs or DW Lyn stars after the prototype (Schuh et al. 2006).

Non-adiabatic computations by Charpinet et al. (1997) predicted excited low degree $(\ell)$, low radial order $(n) p$-modes in sdB models, driven by an iron opacity bump, in the temperature range $29-37 \mathrm{kK}$. This mechanism is inefficient at solar metallicity, but gravitational settling and radiative levitation can work together to locally enhance metals in a driving zone in the envelope. This $\kappa$ mechanism has been successfully invoked to explain both the $p$-mode pulsations in V361 Hya stars and the $g$-mode pulsations in V1093 Her stars (Fontaine et al. 2003). Jeffery \& Saio (2006) were able to expand the instability regions by including opacities for nickel, to the point where the $p$-mode and $g$-mode domains overlap, thereby explaining the presence of hybrid oscillations. However, it is still not understood why most stars in this temperature range do not appear to vary.

As the number of known short-period and hybrid sdBV stars have increased, several peculiar features are emerging:

- Mode density: the number of pulsation frequencies detected vary from a single one to more than 50. LS Dra ( Østensen et al. 2001a) was confirmed to be monoperiodic well below the millimagnitude level from $127 \mathrm{~h}$ of observations by Reed et al. (2007b). For others, detailed follow-up observations have increased the number of detected frequencies to more than 50.

- Period grouping: pulsation periods for the whole V361 Hya group lie between 90 and $800 \mathrm{~s}$, but distinctions appear to be present between the stars in the shorter and longer period groups. All the sdBVs with periods between 300 and $400 \mathrm{~s}$ except one are DW Lyn type hybrid pulsators. The odd one out is KLUMa, which also stands out as the only binary of the pulsators with periods in this range (O'Toole et al. 2004).

- Amplitude variations: amplitudes are found to change with time in many cases. However, it is unclear whether this is due to true amplitude variations or beating of closely spaced modes (Kilkenny 2010).

- Dominant modes: according to their amplitudes the stars can be grouped into high amplitude pulsators, for which a mode was observed to exceed $30 \mathrm{mmag}$ at least once, and low-amplitude ones for which such dominant modes were never reported. All the high amplitude pulsators are DW Lyn type, except the unique V338 Ser, which lies well above the canonical EHB region.

The V361 Hya stars with very few exited modes are not suitable for the period-matching technique in asteroseismology, since too many models can be found that adequately match their sparse periodograms. However, if a mode has sufficient amplitude, the degree $\ell$ can be determined from spectroscopy, substantially constraining the asteroseismic solution. For low-amplitude V361 Hya stars with sufficiently rich spectra, however, asteroseismology has met with great success, as the most fundamental parameter of a star - its mass - has been derived for ten such pulsators along with their fractional hydrogen envelope masses (see Østensen 2009 for a review; and Randall et al. 2009b for the most recent result).
Nevertheless, sdBVs with sparse pulsation spectra are important as they may allow the measurement of light travel time variations or period changes due to stellar evolution, if the modes are sufficiently stable, as exemplified by the planethosting V391 Peg (Silvotti et al. 2007), discovered by our survey (Østensen et al. 2001b). Hence, photometric surveys to discover new V361 Hya stars are still rewarding.

The theoretical location of the sdBV instability strip in the $T_{\text {eff }}-\log g$ plane is quite well established. However, no more than one out of ten sdB stars located within the instability region are actually found to pulsate. This situation is quite different from that of white dwarf pulsators of the ZZ Ceti type, for which evidence is growing that all white dwarfs in their instability strip are pulsating (Gianninas et al. 2007). For sdBVs it has been suggested that younger EHB stars may not have enough iron accumulated in the driving region for the $\kappa$-mechanism to be efficient enough to drive pulsations, but time-dependent diffusion calculations by Fontaine et al. (2006) have demonstrated that sufficient iron for pulsations to occur accumulates after a few hundred thousand years, and after $1 \mathrm{Myr}$ no further accumulation is achieved. Compared to the EHB lifetime of $100-150 \mathrm{Myr}$ this is not significant. An interesting speculation was made by Jeffery \& Saio (2007), that the iron group element enhancements may be disrupted by the atmospheric motions as pulsations build up to some level. They note that since $p$-modes mostly involve vertical motion, while $g$-modes are dominated by horizontal motion, it is possible that $p$-modes are more effective at redistributing the iron group elements out of the driving zone. This could explain the observation that most cool EHB stars are $g$-mode pulsators while at the hotter end of the branch most are not (Green et al. 2003). It may also be that the shortage of pulsators is due to observational biases, either that the pulsations have too low amplitudes, or that they have too high $\ell$ to be easily detectable photometrically, an issue that needs further investigation. To this end it also is important to publish observational constraints on stars that were not found to be variable in order to guide future more sensitive surveys.

Here we present the final results of a long-term programme to detect new short-period sdBVs using the Nordic Optical Telescope (NOT) on La Palma.

\section{The survey}

Shortly after the discovery of the first pulsating sdBs by the South African group (Kilkenny et al. 1997; Koen et al. 1997; Stobie et al. 1997a; O'Donoghue et al. 1997), it was decided to initiate a programme to search for such pulsators using the $2.5 \mathrm{~m}$ NOT on La Palma. The principal aim was to extend the number of sdB pulsators, determine the fraction of variables within this class of stars, and explore the boundaries of their instability region. The South African group had at the time discovered and confirmed pulsations in 14 objects from a sample of around 600 spectroscopically confirmed hot subdwarf stars, and a Canadian team found four pulsators in 74 stars after selecting candidates based on $T_{\text {eff }}$ (Billères et al. 2002). Since those samples were overlapping considerably, two of the pulsators were found in both samples. To avoid searching an already depleted sample, we drew our targets primarily from a new spectroscopic study targeting sdB star candidates from the Hamburg-Schmidt (HS) survey (Hagen et al. 1995), although it was complemented with targets from other surveys as explained below.

The complete list of observational runs fully or partly used for this survey is presented in Table 1 . In Table 5 we provide coordinates and observational limits on all sdB stars checked for 
variability during the survey, except those that were found to pulsate or vary due to eclipses and reflection effects. The eclipsing and reflection variables are published in separate papers, and the 20 pulsators are listed in Table 9, together with pulsators found by other surveys.

\subsection{Chronology and results published to date}

The rapid pulsations in sdBVs are a challenge to observe with standard CCD instrumentation due to the pulsation periods being comparable to the typical readout times of a full CCD frame. An essential part of this project was the design of a system capable of efficient windowed CCD photometry ( $\varnothing$ stensen \& Solheim 2000). A dedicated camera was built for this purpose, the Troms $\varnothing$ CCD Photometer (TCP), and the control system implemented for this camera was adopted to both the HIRAC and ALFOSC cameras at the NOT.

The first search run was made in the summer of 1999 (cf. Table 1), using the Troms $\varnothing$-Texas Photometer (TTP) and revealing the first $\mathrm{sdB}$ pulsator from this programme (Silvotti et al. 2000, Paper I). Already the same autumn the CCD system was operational, and the improved detection efficiency allowed us to reach acceptable noise levels in a much shorter time, thereby resulting in the discovery of three new pulsators in only five nights of observations (Østensen et al. 2001b, Paper II). Two observation runs in 2000 led to the discovery of four more pulsators (Østensen et al. 2001a, Paper III). At this time we started to run out of targets from the HS survey at some RA's, and supplemented our list with stars from the PG survey (Green et al. 1986). This was a concern, as many of the known sdB stars had already been targeted by the South African group, and we expected our detection efficiency to drop since we avoided targeting stars already reported to pulsate. Still, the sample was not completely depleted, and during the summer 2001 run we discovered pulsations in QQ Vir (PG 1325+101) and EP Psc which originated from the HS sample (HS 2303+0152) but also appear in the PG catalogue as PG 2303+019 (Silvotti et al. 2002b, Paper IV).

To get a sample that was both sufficiently large to properly determine the extent of the sdB instability region and the fraction of pulsators across this region, and at the same time undepleted, it was decided to supplement our target lists with stars from the SDSS survey (Stoughton et al. 2002). The first three SDSS stars were observed in October 2002, and one of them, SDSS J171722.10+580559.9 or J1717+5805 for short, was found to pulsate (Solheim et al. 2004, Paper V).

In spite of the initial success of our programme, the Nordic time-allocation committee started turning down our proposals for further search time, and the last regular search run on the original programme was allocated for February 2005, but was completely lost due to bad weather. The last successful search run was made in June 2004, resulting in three new pulsators. These are PG 1419+081, SDSS J144514.93+000249.0 $(\mathrm{J} 1445+0002)$ and SDSS J164214.21+425234.0 (J1642+4552). Only brief sequences were made on these objects, and the results were presented in Solheim \& Østensen (2006, Paper VI).

At this time a collaborative search was initiated with a Spanish team (RO, AU, FPH, CRL), which permitted additional access to the NOT through Spanish time. With the Spanish group, we were able to explore a larger number of stars drawn from the Subdwarf Database (Østensen 2004, 2006). The first pulsator discovered with the NOT on Spanish time was another star drawn from the SDSS sample PG 1657+416 (SDSS J165841.83+413115.6), and was presented by
Table 1. Observation runs at the Nordic Optical Telescope contributing to this survey.

\begin{tabular}{|c|c|c|c|c|}
\hline ID & Dates & $N_{n}$ & $N_{t}$ & Observers \\
\hline N1 & 1999 July 19-23 & 4.0 & 13 & JES, RS, JMGP \\
\hline $\mathrm{N} 2$ & 1999 Oct. $14-19$ & 5.0 & 31 & JES, RHØ \\
\hline N3 & 2000 July 7-11 & 4.0 & 25 & RHØ, RS \\
\hline N4 & 2000 Oct. 4-8 & 4.0 & 38 & JES, RHØ \\
\hline N5 & 2001 July 25-29 & 4.0 & 22 & RHØ, RS \\
\hline N6 & 2002 Oct. $10-15$ & 0.5 & 4 & $\mathrm{JES}^{a}$ \\
\hline N7 & 2004 June $4-8$ & 4.0 & 44 & JES, RHØ \\
\hline No & 2005 Feb. $15-20$ & 5.0 & 0 & $\mathrm{JES}^{b}$ \\
\hline N8 & 2006 Dec. 10-15 & 0.3 & 4 & $\mathrm{JES}^{a}$ \\
\hline N9 & 2007 Dec. 14-16 & 3.0 & 14 & $\mathrm{RH}, \mathrm{JHT}^{c}$ \\
\hline $\mathrm{X} 1$ & 2004 Oct. 15-17 & 0.3 & 4 & JES, RH $\emptyset^{d}$ \\
\hline $\mathrm{S} 1$ & 2004 May 21-24 & 4.0 & 20 & $\mathrm{RO}$ \\
\hline $\mathrm{S} 2$ & 2005 May 30-June 1 & 4.0 & 15 & RO \\
\hline S3 & 2007 Oct. 20-24 & 3.0 & 11 & RO \\
\hline S4 & 2008 Sep. 22-25 & 3.0 & 20 & RO \\
\hline S5 & 2008 July 26-30 & 2.0 & 67 & RH $\emptyset^{e}$ \\
\hline S6 & 2009 Jan. 24-27 & 3.0 & 5 & RO \\
\hline $\mathrm{T} 1$ & 2001 Feb. 24 & 0.6 & 7 & RH $\varnothing^{f}$ \\
\hline $\mathrm{T} 2$ & 2001 April 10, 13 & 2.0 & 24 & RH $\varnothing^{e}$ \\
\hline T3 & 2002 Sep. 13-14 & 0.2 & 4 & RHØ, $\mathrm{JHT}^{f}$ \\
\hline $\mathrm{T} 4$ & 2003 Feb. 10-19 & 0.5 & 5 & RHØ, $\mathrm{JHT}^{f}$ \\
\hline T5 & 2004 Jan. 13 & 0.5 & 9 & RHØ, $\mathrm{JHT}^{f}$ \\
\hline T6 & 2005 May 5 & 0.7 & 16 & RH $\emptyset^{f}$ \\
\hline $\mathrm{T} 7$ & 2006 May 9 & 0.7 & 15 & $\mathrm{RH} \emptyset^{f}$ \\
\hline T8 & 2008 Feb. 24, 26 & 0.2 & 2 & RH $\varnothing^{f}$ \\
\hline ST & 2006 June 10 & 0.5 & 2 & $\mathrm{JMGP}^{g}$ \\
\hline Total & & 59.0 & 421 & \\
\hline
\end{tabular}

Notes. The ID column gives our run identifier, used to refer to a particular observation run in Table 5 and when discussing particular observations. $N_{\mathrm{n}}$ gives the number of nights used for this survey programme. For nights allocated to the survey, the number includes all nights, regardless of whether any observations were actually made or not. For nights where this programme served as a backup for another, only the fraction of nights actually used for this programme is listed. The $N_{\mathrm{t}}$ column shows the number of targets observed. Note that reobservations and observations of sdO stars (to be analysed in a separate paper) are included in these totals. The notes indicate.

(a) Run primarily dedicated to an unrelated target.

(b) Run lost due to snow and ice.

(c) Run primarily for follow-up of our pulsators.

(d) Xcov 24 multi-site campaign.

(e) Run primarily dedicated to search for sdO stars.

(f) Run primarily for technical investigations.

(g) Spanish service time.

Oreiro et al. (2007, Paper VII). Observation runs allocated on Nordic time are indicated by run numbers prefixed by $\mathrm{N}$, and on Spanish time by $\mathrm{S}$ in Table 1.

Nordic time still continued to be granted for follow-up programmes on various pulsating stars, including one of the most interesting pulsators discovered during this programme, V391 Peg, which was demonstrated to be host to a planet by Silvotti et al. (2007), by using the orbital period modulation introduced on the two main pulsation modes. A few unexplored pulsator candidates were observed during these runs (N6, N8, N9 in Table 1), and also during the WET XCOV 24 campaign on the pulsating sdB star UY Sex (Vučković et al. 2006, run X1), whenever the prime target was not reachable. Upgrades and tests on the fast CCD photometry system also gave several opportunities to observe some additional targets during technical time at the NOT (listed as T1 to T8 in Table 1). One new pulsator, PG 1033+201, was found during run $\mathrm{T} 7$. 
The limitations on telescope time from 2004 onward forced us to abandon the faint SDSS targets. Instead, we focused on detecting more pulsators in the cool region where the particularly interesting objects V391 Peg and Balloon 090100001 had been found. The discovery of hybrid pulsations in DW Lyn by Schuh et al. (2006) provided further encouragement for increasing the sample in this region of the instability strip. Although no more such cool pulsators were detected, a total of five more pulsators in the hotter end of the instability strip were found. One, from the BG sample (Bok-Green, Green et al. 2008), was recently published in Oreiro et al. (2009, Paper VIII), and four are presented for the first time in this paper.

In addition to these 20 pulsators our photometric observations have revealed several of the relatively rare $\mathrm{sdB}$ stars with M-dwarf companions from their strong reflection effect. The discovery of an eclipsing sdB $+\mathrm{dM}$ system, HS 0705+6700, first detected during run N4 was presented by Drechsel et al. (2001), and the non-eclipsing sdB+dM system HS 2333+3927 discovered during run N2 was presented by Heber et al. (2004). Two more sdBs with $\mathrm{dM}$ companions were found during run S2: the eclipsing HS 2231+2441 (Østensen et al. 2007), and HS 2043+0615 (Østensen et al. 2010, A\&A, in prep.).

Our survey was not originally designed to detect long period variability, since we aimed for short photometric sequences with high $S / N$. However, the large photometric amplitudes of $\mathrm{sdB}+\mathrm{dM}$ binaries produce a strong trend in the differential photometry, of which we saw several examples. However, since follow-up of such long-period variable objects can easily be done with smaller telescopes using long integration times, no follow-up was attempted on these stars with the NOT. The long period $g$-mode sdBVs also pulsate on time-scales around one hour, but with much lower amplitudes. As they had yet to be discovered at the start of our survey, our observation strategy was not designed with these objects in mind.

\subsection{The HS/HE/SPY samples}

When our programme was initiated, the main source of targets was the HS survey, but we also included targets from the equatorial part of the Hamburg-ESO (HE) survey (Wisotzki et al. 1996). Follow-up spectroscopy made at Calar Alto with the TWIN spectrograph (Heber et al. 1999; Edelmann et al. 2003) allowed us to preselect candidates with effective temperatures and gravities in the domain predicted for the pulsational instability (Charpinet et al. 1997). This provided us with a sample of pulsator candidates that was both tuned to the instability region and relatively undepleted by previous surveys. The latter was particularly important since the South African group had already explored a considerable fraction of the sdB stars known in the literature (and compiled in the Catalogue of spectroscopically identified hot subdwarf stars, Kilkenny et al. 1988). However, it has turned out that the HE survey has a significant overlap with unpublished parts of the Edinburgh-Cape (EC) blue object survey (Stobie et al. 1997b), which was one of the main sources surveyed by the South African group. This can explain why out of 80 stars from the HS survey and 20 stars from the HE survey observed during the initial five runs (as reported in Paper V), pulsations were found in eight HS stars and in none of the HE stars. Due to the difficulty of establishing accurate effective temperatures and gravities for $\mathrm{sdB}+\mathrm{F}-\mathrm{K}$ stars, these were not included in our initial sample, and were only added later when the priority sample started to become depleted. Thus, unlike the South African group, who found that most of their first pulsators were in binary systems, due to the selection biases involved only one
Table 2. Analysis for eight stars from the HS survey, not included in Edelmann et al. (2003).

\begin{tabular}{lccccc}
\hline \hline Name & $\begin{array}{c}m_{\mathrm{pg}} \\
\mathrm{mag}\end{array}$ & Class & $\begin{array}{c}T_{\mathrm{eff}} \\
\mathrm{kK}\end{array}$ & $\begin{array}{c}\log g \\
\mathrm{dex}\end{array}$ & $\begin{array}{c}\log y \\
\mathrm{dex}\end{array}$ \\
\hline Observed with CA/TWIN & & & & \\
HS 1733+4540 & 15.1 & $\mathrm{sdOB}$ & 37.5 & 5.6 & -3.0 \\
HS 1909+7004 & 15.4 & $\mathrm{sdB}$ & 36.5 & 5.4 & -2.9 \\
Spectra courtesy of & Aguilar-Sánchez & $(1998)$ & & \\
HS 0026+0439 & 14.8 & $\mathrm{sdB}$ & 36.7 & 5.8 & -3.0 \\
HS 0042+0927 & 16.0 & $\mathrm{sdB}$ & 30.2 & 5.6 & -2.9 \\
HS 2254+0640 & 15.2 & $\mathrm{sdB}$ & 29.1 & 5.6 & -2.7 \\
HS 2320+0840 & 14.6 & $\mathrm{sdB}+$ & 30.0 & 5.8 & -2.6 \\
HS 2323+0459 & 14.8 & $\mathrm{sdB}$ & 29.3 & 5.4 & -2.8 \\
HS 2334+0144 & 15.5 & $\mathrm{sdB}$ & 30.8 & 5.6 & -2.6 \\
\hline
\end{tabular}

Notes. $m_{\mathrm{pg}}$ gives the photographic magnitude from the HS survey plates, "Class" our spectroscopic classification, followed by the effective temperature $\left(T_{\text {eff }}\right)$, surface gravity $(\log g)$, and atmospheric helium abundance $\left(\log y=\log \left(N_{\mathrm{He}} / N_{\mathrm{H}}\right)\right)$, from our model fits. The $\mathrm{sdB}+$ classification indicates a composite spectrum. The notes on the names imply: ${ }^{(1)}$ Also found in the PG survey (Green et al. 1986).

(2) Also found in the KUV survey (Noguchi et al. 1980).

of our initial 10 were. Note that another composite, our first pulsator LM Dra, is in a well-separated visual binary with an F3 companion, and therefore not considered to be an $\mathrm{sdB}+\mathrm{F}$ system since the stars cannot have interacted during their evolution.

Only eight stars from the HS sample observed during this survey have not yet been published elsewhere. Their coordinates are listed in the on-line table together with those of all the other targets (Table 5), and their physical parameters are listed in Table 2. We computed the stellar atmospheric parameters (effective temperature, surface gravity, and photospheric helium abundance) by fitting model atmosphere grids to the hydrogen and helium lines visible in the spectra. The procedure used for this fitting was the same as that of Edelmann et al. (2003), but using only the LTE models described in Heber et al. (2000), as the purpose of our modelling was only to establish which stars are roughly within the sdB instability region rather than to establish precise physical parameters.

The parameters of HS $1909+7004$ are uncertain since the spectrum is of low resolution and the fit is quite poor, but the fits to the other stars appear to be quite good. HS $2320+0840$ has a very strong companion that contributes clearly visible line features in the spectrum. For this reason, we performed a spectral decomposition, subtracting a main sequence model from Munari et al. (2005) with $T_{\text {eff }}=6.5 \mathrm{kK}, \log g=4$ and solar metallicity, which we found to contribute about $40 \%$ of the flux at $5000 \AA$.

A total of 46 objects from the HE survey were observed. For 23 of these the physical parameters were determined by Edelmann (2003), and none of these were found to pulsate. Cross-referencing them with the literature reveals that only nine are new, the rest are subdwarfs with identifications from the Montreal-Cambridge-Tololo (MCT) Survey (Lamontagne et al. 2000), or earlier surveys compiled by Kilkenny et al. (1988).

A number of white dwarf candidates from the HE survey were observed as part of the SPY survey (Napiwotzki et al. 2001) and published by Lisker et al. (2005), where physical parameters are provided also for the composite objects. $23 \mathrm{HE}$ stars from the SPY sample were observed, and two were found to pulsate. Also, four HS stars from this sample were observed, and one more pulsator was found. These three new variables are presented in Sect. 5. Lisker et al. (2005) also present a list of sdB stars 
formerly misclassified as white dwarfs. We observed four of these, but did not detect signs of variability ${ }^{3}$.

\subsection{The SDSS sample}

More than 16000 spectra of UV excess stars were downloaded from the SDSS survey (Stoughton et al. 2002) up to data release 6 (DR6), and the hot subdwarfs were identified by inspection. The intermediate resolution $(\sim 1 \AA)$ SDSS spectra cover all wavelengths from 3800 to $9000 \AA$, ideal for classification purposes. We identified about 900 objects as hot subdwarf stars and classified them into sdB, sdO, sdOB, He-sdB, He-sdO, and He-sdOB, depending on whether hydrogen or helium dominates the spectrum, and if they contain lines from He I, He II or both. 318 of the sdB stars were classified as single sdB stars and 165 as sdB $+F-K$ binaries $(34 \%)$ based on spectroscopic signatures and their position in $\left(u^{\prime}-g^{\prime}, g^{\prime}-r^{\prime}\right)$ colour-colour space. A similar fraction was found for the sdOB stars; 78 single and 35 binaries (31\%). All these stars were fitted to model spectra to determine their position in the $T_{\text {eff }} / \log g$ plane, using the Balmer lines $\mathrm{H} \alpha$ to $\mathrm{H} \eta$, as well as the most prominent helium lines at $4472 \AA$ and $4026 \AA$ for the sdB stars, and including He II 4686 for the sdOBs. The formal fitting errors are about $250 \mathrm{~K}$ for $T_{\text {eff }}, 0.05 \mathrm{dex}$ for $\log g$, and $0.1 \mathrm{dex}$ for $\log y=\log \left(N_{\mathrm{He}} / N_{\mathrm{H}}\right)$ at a $g^{\prime}$ magnitude around 16.0 , but increase by a factor of two for the fainter stars between $g^{\prime}=17.5$ and 18 . For the stars with F-G companions the uncertainties are larger, but not so large that the parameters are not useful for establishing whether or not the stars are located within the instability region. We note also that for the hottest stars in the sample, NLTE effects start to become significant (Napiwotzki 1997), and that the LTE models can underestimate the temperatures by up to $1000 \mathrm{~K}$ in some cases.

Only 40 stars from this large sample were observed due to observing time restrictions. Five were found to pulsate and are listed in Table 4 with additional details in Table 9. The remaining 35 objects are listed in Table 3 , with the physical parameters we derived from the SDSS archive spectra. The sample includes eleven spectroscopic binaries, and curiously all five pulsators found from the SDSS sample were among these. The physical parameters for the binaries have considerable systematic shifts depending on the contribution from the main sequence companion. For this reason we have made a spectral decomposition of the five pulsators in order to place them reliably in the $T_{\text {eff }} / \log g$ plane. The details of the spectroscopic decomposition are given in Table 4 . The six non-pulsating $\mathrm{sdB}+\mathrm{F}-\mathrm{K}$ systems were fitted as if they were single stars.

It is non-trivial to estimate the spectroscopic parameters of the companion, but a reasonable compromise can usually be made that approximates the spectral contributions from the main sequence companion to the $\mathrm{Ca}$ II $\mathrm{H}$ and $\mathrm{K}$ lines as well as the $g$-band and the $\mathrm{MgI}$ lines. For the spectral decomposition we used the SDSS spectra processed with the DR7 pipeline, which have a significantly improved flux calibration compared with earlier data releases. After dereddening the spectrum by the $E(B-V)$ reddening coefficients provided by the Schlegel et al. (1998) dust maps, the flux was fitted with one of our subdwarf model spectra and a main sequence model spectrum from Munari et al. (2005). The parameters of the subdwarf are then refitted to the observed spectrum after subtraction of the main sequence model, and the procedure was iterated until it

\footnotetext{
3 Actually, one more of the fourteen stars in this list of misclassified WDs is included in our sample: Ton S 155 appears in Edelmann (2003) as HE 0021-2326.
}

Table 3. The stars from the SDSS survey observed during this programme, excluding the pulsators listed in Table 4.

\begin{tabular}{|c|c|c|c|c|c|}
\hline Name & $\begin{array}{c}g^{\prime} \\
\mathrm{mag}\end{array}$ & Class & $\begin{array}{l}T_{\text {eff }} \\
\mathrm{kK}\end{array}$ & $\begin{array}{l}\log g \\
\operatorname{dex}\end{array}$ & $\begin{array}{l}\log y \\
\operatorname{dex}\end{array}$ \\
\hline PB $5916^{1}$ & 15.3 & $\mathrm{sdB}$ & 30.1 & 5.7 & -2.3 \\
\hline PG 0812+482 & 15.0 & $\mathrm{sdB}$ & 25.0 & 5.4 & -3.0 \\
\hline PG $0826+480^{2}$ & 15.8 & $\mathrm{sdB}$ & 26.1 & 5.5 & -3.1 \\
\hline PG $1100-008^{2}$ & 16.3 & $\mathrm{sdB}+$ & 29.2 & 5.5 & $-2.6^{\dagger}$ \\
\hline PG $1136-003^{1,2}$ & 14.2 & $\mathrm{sdB}$ & 31.6 & 5.6 & -2.7 \\
\hline PG $1249+028^{1,2,3}$ & 15.4 & $\mathrm{sdB}$ & 30.6 & 5.6 & -3.0 \\
\hline $\mathrm{J} 12596-0039^{4}$ & 16.8 & $\mathrm{sdOB}+$ & 34.4 & 5.6 & $-2.2^{\dagger}$ \\
\hline PG $1315+013^{1,2}$ & 16.6 & $\mathrm{sdB}$ & 26.6 & 5.3 & -2.3 \\
\hline $\mathrm{J} 13320+6733^{4}$ & 17.0 & sdOB & 36.1 & 6.0 & -1.1 \\
\hline $\mathrm{J} 13516+0234^{1}$ & 17.1 & $\mathrm{sdB}+$ & 34.7 & 5.3 & $-3.0^{\dagger}$ \\
\hline PG $1403+019^{1,2}$ & 15.6 & $\mathrm{sdB}$ & 27.7 & 5.5 & -1.9 \\
\hline $\mathrm{J} 14086+6531^{1}$ & 17.4 & $\mathrm{sdB}$ & 30.4 & 5.6 & -3.0 \\
\hline $\mathrm{J} 14236+0149^{1}$ & 17.1 & $\mathrm{sdB}$ & 29.5 & 5.7 & -2.3 \\
\hline PG $1422+035^{2}$ & 16.2 & sdOB & 35.1 & 5.8 & -1.5 \\
\hline $\mathrm{J} 15107+0409^{4}$ & 16.9 & sdOB & 34.7 & 5.7 & -1.6 \\
\hline $\mathrm{J} 15131+0114^{1}$ & 17.1 & $\mathrm{sdB}$ & 28.0 & 5.6 & -3.0 \\
\hline EGGR $491^{1,5}$ & 16.8 & $\mathrm{sdB}$ & 30.6 & 5.6 & -3.0 \\
\hline SBSS $1544+568^{1}$ & 16.8 & $\mathrm{sdB}$ & 27.2 & 5.2 & -2.0 \\
\hline $\mathrm{J} 15481-0049^{1}$ & 16.4 & $\mathrm{sdB}+$ & 32.7 & 5.6 & $-3.0^{\dagger}$ \\
\hline $\mathrm{J} 15513+0649$ & 15.9 & sdOB+ & 34.3 & 5.0 & $-2.1^{\dagger}$ \\
\hline $\mathrm{J} 15564+0113^{1}$ & 16.0 & $\mathrm{sdB}$ & 30.7 & 5.7 & -3.1 \\
\hline $\mathrm{J} 16026-0012^{1}$ & 17.5 & $\mathrm{sdB}$ & 34.0 & 5.8 & -2.1 \\
\hline $\mathrm{J} 16165-0038^{1}$ & 16.7 & sdOB & 36.0 & 5.7 & -1.8 \\
\hline $\mathrm{J} 16331+0032^{1}$ & 16.9 & $\mathrm{sdB}$ & 31.7 & 5.8 & -3.3 \\
\hline $\mathrm{J} 16347-0053^{1}$ & 16.9 & $\mathrm{sdB}$ & 29.1 & 5.5 & -2.7 \\
\hline $\mathrm{J} 16420+4403^{1}$ & 16.7 & $\mathrm{sdB}$ & 28.8 & 5.2 & -2.8 \\
\hline $\mathrm{J} 16443+4523^{1}$ & 17.1 & $\mathrm{sdB}$ & 33.1 & 5.8 & -2.0 \\
\hline PG $1653+633^{1,2}$ & 15.9 & sdOB & 36.1 & 5.9 & -1.5 \\
\hline $\mathrm{J} 17144+6147^{1}$ & 16.6 & $\mathrm{sdB}+$ & 33.4 & 5.6 & $-3.4^{\dagger}$ \\
\hline SBSS $1715+556^{1,6}$ & 16.9 & $\mathrm{sdB}$ & 30.3 & 5.4 & -3.2 \\
\hline $\mathrm{J} 17165+5751^{4}$ & 18.0 & $\mathrm{sdOB}$ & 34.9 & 5.8 & -0.8 \\
\hline $\mathrm{J} 20440-0511^{1}$ & 17.3 & $\mathrm{sdB}$ & 29.9 & 5.4 & -3.2 \\
\hline $\mathrm{J} 20457-0543^{1}$ & 17.8 & $\mathrm{sdB}$ & 34.9 & 5.4 & -1.7 \\
\hline $\mathrm{J} 20573-0626^{1}$ & 16.5 & $\mathrm{sdB}$ & 28.0 & 5.1 & -2.5 \\
\hline $\mathrm{J} 21531-0719^{1}$ & 16.9 & $\mathrm{sdB}$ & 32.0 & 5.9 & -2.0 \\
\hline
\end{tabular}

Notes. The columns are as in Table 2, except that Sloan $g^{\prime}$ magnitudes are provided provided as indicator of the signal level. A plus sign after the class marks the presence of a cool companion from features in the spectrum, the slope of the spectrum or $z$-band excess. The notes indicate:

(1) Correctly identified as sdB stars in Eisenstein et al. (2006).

(2) Classified as hot subdwarf stars in Green et al. (1986).

(3) Object misnamed in the PG catalog; should be PG 1249-027.

(4) Listed as sdO stars in Eisenstein et al. (2006).

(5) Misclassified as a white dwarf in McCook \& Sion (1987).

(6) Identified as an sdB star in Stepanian et al. (2001).

(†) Parameters for composites are less certain than for singles.

converged. Note that the reddening coefficients are for lines of sight to infinity and that we have no guarantee that the particular subdwarf under consideration is not in front of the bulk of the dust. There is also a significant uncertainty in the $E(B-V)$ values due to the limited resolution of the Schlegel et al. (1998) maps and the lumpiness of the interstellar medium. But due to the low resolution and limited signal in the SDSS spectra, it is hard to make a complete disentanglement of the spectra while leaving the reddening as a free parameter, so we will stick with the dust map values. In the case of $\mathrm{J} 1445+0002$, which has the highest reddening coefficient of the five, it is quite clear that the $\mathrm{Ca}$ II lines are too narrow to originate from the main 
Table 4. Spectral decomposition of the binary pulsators from the SDSS sample.

\begin{tabular}{|c|c|c|c|c|c|c|c|c|c|c|c|c|c|}
\hline \multirow[b]{2}{*}{ Name } & \multicolumn{4}{|c|}{ Companion signature } & \multirow[b]{2}{*}{$\begin{array}{l}E(B-V) \\
\text { mag }\end{array}$} & \multicolumn{4}{|c|}{ Primary } & \multicolumn{4}{|c|}{ Secondary } \\
\hline & $\begin{array}{l}\mathrm{Ca} \text { II } \\
\mathrm{H}+\mathrm{K}\end{array}$ & $\begin{array}{c}\mathrm{CH} \\
\mathrm{G}\end{array}$ & $\underset{\mathrm{b}}{\mathrm{Mg}}$ & $\begin{array}{c}\mathrm{Na} I \\
\mathrm{D}\end{array}$ & & $f_{5000}$ & $\begin{array}{l}T_{\text {eff }} \\
\mathrm{kK}\end{array}$ & $\begin{array}{l}\log g \\
\operatorname{dex}\end{array}$ & $\log y$ & $f_{5000}$ & $\begin{array}{l}T_{\text {eff }} \\
\mathrm{kK}\end{array}$ & $\begin{array}{l}\log g \\
\operatorname{dex}\end{array}$ & {$[\mathrm{M} / \mathrm{H}]$} \\
\hline $\mathrm{J} 1717+5805$ & $\sqrt{ }$ & & $\sqrt{ }$ & $\sqrt{ }$ & 0.025 & 0.90 & 34.4 & 5.75 & -1.8 & 0.10 & 5.0 & 4.0 & +0.0 \\
\hline PG $1657+416$ & $\sqrt{ }$ & $\sqrt{ }$ & $\sqrt{ }$ & $\sqrt{ }$ & 0.021 & 0.74 & 32.2 & 5.80 & -2.0 & 0.25 & 5.5 & 4.0 & +0.0 \\
\hline PG $1419+081$ & $\sqrt{ }$ & $\sqrt{ }$ & $\sqrt{ }$ & $\sqrt{ }$ & 0.028 & 0.86 & 33.3 & 5.85 & -1.8 & 0.14 & 5.5 & 4.0 & +0.0 \\
\hline $\mathrm{J} 1445+0002$ & $\sqrt{ }$ & & $\sqrt{ }$ & $\sqrt{ }$ & 0.11 & 0.83 & 35.9 & 5.75 & -1.6 & 0.17 & 6.0 & 4.0 & +0.0 \\
\hline $\mathrm{J} 1642+4552$ & $\sqrt{ }$ & $\sqrt{ }$ & $\sqrt{ }$ & $\sqrt{ }$ & 0.011 & 0.53 & 32.4 & 5.80 & -2.0 & 0.47 & 6.0 & 4.0 & -1.5 \\
\hline
\end{tabular}

sequence companion, and no solution can be found that removes the CaII contribution from the subdwarf spectrum. Therefore, the Ca II signature is mostly interstellar in origin, consistent with the high reddening value. The strongest companion by far is that of J1642+4552, in which it contributes almost half the light at $\lambda=5000 \AA$ ( $f_{5000}$ in Table 4$)$. The system is also in a region of very low extinction, reducing the ambiguity of the spectral decomposition. The strong companion and good signal in this particular SDSS spectrum allow us to make some further constraints on the companion. It is clear that a solar metallicity makes a far stronger contribution to the metal bands than allowed by the observed spectrum. A better fit is achieved with a spectrum metal depleted relatively to solar by $[\mathrm{M} / \mathrm{H}]=-1.5$.

The temperatures derived here are somewhat lower than the temperatures we originally used to select the stars, but not enough to make a difference with respect to the sample selection, as we suspected (Paper VII). For J1445+0002 the temperature drops from 37.6 to a more reasonable $35.9 \mathrm{~K}$. It is suspicious that the derived $\log g$ values for all the five composite stars are so similar (within $\pm 0.1 \mathrm{dex}$ ), and might be due to degeneracies when fitting so many free parameters to spectra with rather low resolution. It has also been noted that more reliable estimates of the surface gravity are obtained when the spectra cover all the high order Balmer lines, a requirement the SDSS spectra do not satisfy.

\subsection{The Bok-Green sample}

Eleven stars in our survey were selected from a spectroscopic campaign to obtain a large unbiased sample of hot stars based on 2MASs colours, undertaken by Green et al. (2008) with the University of Arizona $2.3 \mathrm{~m}$ Bok telescope. Physical parameters were derived from a subsample of 89 stars from this campaign by Winter (2006, Appendix C). None of the stars we observed have reliable spectroscopic classifications in the literature, but three have been listed as faint blue stars by earlier surveys (Ton 930, FBS 1133+754, and FBS 1224+780). However, none of these were known to be hot subdwarf stars, so we consider all eleven as belonging to an undepleted sample. One of the sdBs in the BG sample was found to pulsate, $2 \mathrm{M} 0415+0154=2 \mathrm{MASS}$ J04155016+0154209, as reported in Paper VIII. The remaining seven stars are listed with $2 \mathrm{M}$ designation in Table 5.

\subsection{Literature}

To supplement our sample with targets to fill all RAs we added stars with temperatures and gravities published in the literature. Most of these additional objects are well-known subdwarfs from the PG survey and are included in many studies. The largest sample used was that of Saffer et al. (1994), where 24 targets were surveyed and one found to pulsate (LM Dra).
Seventeen PG stars from the radial velocity survey of Maxted et al. (2001), and four stars from the HST study of Heber et al. (2002) were also included in our sample, but none were found to be variable.

Rough temperature estimates can also be made on the basis of photometric measurements alone, and several works provide such estimates. The survey of Beers et al. (1992) includes temperatures computed from UBV colours, and we observed eight of these stars. A handful of composite $\mathrm{sdB}+\mathrm{MS}$ stars from the list of Allard et al. (1994), where their estimate of the temperature for the primary lie within the instability region, were also observed. Another four stars were targeted based on the temperature estimates of Bixler et al. (1991). Similarly, we observed stars from the sample of Moehler et al. (1990), after estimating the temperatures based on their Strömgren photometry and the spectral features visible in their spectroscopic atlas. In total, about fifty stars in the sample were observed based on such photometric temperature estimates, and only one, PG 1419+081, was found to vary. The SDSS spectroscopy of this object was released after our discovery, allowing us to refine the original colour temperature estimate and correctly identify the star as an $\mathrm{sdB}+\mathrm{G} 5$ composite. We found that the colour temperature $\left(T_{\text {eff }}=33.8 \mathrm{kK}\right.$; Beers et al. 1992) is encouragingly close to our model fit value $\left(T_{\text {eff }}=33.3 \mathrm{kK}\right)$.

If we look at the complete sample from Beers et al. (1992) and consider only the stars classified as sdB with temperatures above $27.5 \mathrm{kK}$ we are left with 23 stars, of which we have observed nine and found one variable. But EC 203381925 is also in this sample and was in our original list, although excluded after pulsations were discovered by the South African team (published by Kilkenny et al. 2006, but preliminary results from the 1998 discovery were distributed much earlier). Another star in the sample is BPS CS 22169-1, analysed by Geier et al. (2010, A\&A, in prep.) and found to have $T_{\text {eff }}=39.1 \mathrm{kK}$, much hotter than the $33.9 \mathrm{kK}$ estimate by Beers et al. (1992), and hotter than the predicted sdBV instability region. The star BPS CS 22890-94 = PG 1525+024 was not observed since the colour temperature estimate by Beers et al. (1992) placed it below our cut at $T_{\text {eff }}=22.9 \mathrm{kK}$. However, recent SDSS spectroscopy gives us $T_{\text {eff }}=27.9 \mathrm{kK}$, which would have placed the object inside our cuts, further emphasising the uncertainties associated with using colours as a temperature estimator.

\section{Observations}

During our observations we systematically aimed at identifying as many clear pulsators as possible, rather than trying to push the limit on the pulsation amplitudes as low as possible. Thus, most of our detected variables have rather high pulsation amplitudes, and the sample may still contain objects with undetected amplitudes below $2 \mathrm{mma}$. Due to the shortness of our typical 
R. H. Østensen et al.: A survey for pulsating sdB stars

Table 5. Truncated version of the catalogue of sdB stars observed during our search programme.

\begin{tabular}{|c|c|c|c|c|c|c|c|c|c|c|}
\hline Target Name & Other Name & $\begin{array}{c}\text { RA } \\
\text { J2000 }\end{array}$ & $\begin{array}{c}\text { Dec } \\
\text { J2000 }\end{array}$ & $\begin{array}{l}m_{\mathrm{pg}} \\
\mathrm{mag}\end{array}$ & Run & $N_{\mathrm{p}}$ & $\begin{array}{r}\Delta t \\
\mathrm{~s}\end{array}$ & $\begin{array}{r}\sigma \\
\mathrm{mma}\end{array}$ & $\begin{array}{l}A_{\max } \\
\mathrm{mma}\end{array}$ & Var \\
\hline SB 7 & MCT 0000-1637 & $00: 03: 24.37$ & $-16: 21: 06.3$ & 12.7 & S5 & 60 & 20 & 0.60 & 1.26 & NOV \\
\hline HE 0002-2648 & МСТ 0002-2648 & 00:05:09.58 & $-26: 31: 48.4$ & 15.6 & S5 & 39 & 20 & 1.01 & 2.07 & $\mathrm{NOV}$ \\
\hline HE 0004-2737 & МСТ 0004-2737 & $00: 06: 46.26$ & $-27: 20: 53.4$ & 13.7 & N4 & 60 & 20 & 0.44 & 0.95 & NOV \\
\hline HE 0007-2212 & МСТ 0007-2737 & 00:09:45.91 & $-21: 56: 14.4$ & 14.9 & S5 & 43 & 10 & 0.79 & 1.48 & NOV \\
\hline MCT 0008-1245 & $\ldots$ & 00:11:25.04 & $-12: 28: 45.9$ & 14.3 & S4 & 93 & 15 & 0.59 & 1.04 & NOV \\
\hline KUV $04233+1502$ & $\ldots$ & 04:26:08.00 & $+15: 08: 29.01$ & 14.3 & S4 & 129 & 20 & 0.33 & 1.01 & \\
\hline KPD $0716+0258$ & $\ldots$ & $07: 18: 57.85$ & $+02: 53: 14.67$ & 14.7 & $\mathrm{~T} 4$ & 270 & 30 & 0.25 & 0.78 & \\
\hline KUV 07301+3659 & $\ldots$ & $07: 33: 25.62$ & $+36: 52: 57.83$ & 14.8 & S4 & 134 & 18 & 0.30 & 0.93 & \\
\hline PG $1248+164$ & $\ldots$ & $12: 50: 50.23$ & $+16: 10: 03.40$ & 14.0 & $\mathrm{~T} 7$ & 65 & 20 & 5.07 & 16.61 & \\
\hline PG $1313+132$ & $\ldots$ & $13: 15: 58.16$ & $+12: 57: 40.42$ & 14.1 & S6 & 96 & 20 & 0.95 & 3.35 & \\
\hline $\mathrm{J} 1351+0234$ & $\ldots$ & $13: 51: 40.69$ & $+02: 34: 29.21$ & 17.1 & N7 & 70 & 30 & 0.91 & 2.93 & \\
\hline PG $1722+286$ & $\ldots$ & $17: 24: 11.97$ & $+28: 35: 26.90$ & 12.8 & N1 & 1191 & 5 & 1.01 & 3.08 & \\
\hline PG $1725+252$ & $\ldots$ & $17: 27: 57.39$ & $+25: 08: 35.70$ & 12.7 & $\mathrm{~T} 2$ & 61 & 10 & 3.38 & 12.39 & \\
\hline HS 2029+0301 & $\ldots$ & $20: 31: 52.63$ & $+03: 11: 17.39$ & 15.6 & N1 & 952 & 5 & 2.13 & 6.41 & \\
\hline KPD $2215+5037$ & $\ldots$ & $22: 17: 20.62$ & $+50: 52: 58.09$ & 13.6 & N5 & 45 & 25 & 0.69 & 2.39 & \\
\hline
\end{tabular}

Notes. The full table with 285 entries is available in electronic form from at the CDS. Only the first five entries in the table are listed, plus the ten stars that have peaks between $3 \sigma$ and the $3.7 \sigma$ cutoff required for the NOV flag (discussed in Sect. 4.1). The stars in the sample for which pulsations were detected are listed in Table 9. The columns include target names, coordinates, a magnitude estimate (usually photographic), the run ID from Table 1, the number of data points in the light-curve $\left(N_{\mathrm{p}}\right)$ used to compute the FT, the sampling interval $(\Delta t)$, the mean amplitude in the FT $(\sigma)$, the amplitude of the highest peak in the FT $\left(A_{\max }\right)$, and a non-variability flag.

photometric sequences, there may also be multiperiodic pulsators among our null-detections, unidentified simply because their periods happened to be canceling each other out in a beat phase at the short time they were observed. The stars in Table 5 should therefore not be considered non-variable, rather, we prefer the term not observed to vary (NOV).

For the aims of this campaign we consider a star as NOV if there is no peak in its Fourier Transform (FT) with an amplitude higher than 3.0 times the mean amplitude level $(\sigma)$. However, we require an amplitude of $3.7 \sigma$ (Kuschnig et al. 1997, 99\% confidence level) in order to call the object a pulsator. Objects that fall between these two tiers are considered pulsator candidates until further observations can confirm the detection. Most pulsator candidates were reobserved in subsequent runs, but 11 objects remain in Table 5 with this classification, and will be discussed separately in Sect. 4.1.

Only the frequency region $1.5-10 \mathrm{mHz}$ was used to compute $\sigma$ homogeneously. The low frequency range was excluded to avoid the higher mean amplitude level caused by small trends in the light-curve due to extinction effects or sky transparency variations.

\subsection{PMT photometry}

The first observation run (N1) was made with the Tromsø-Texas 3-channel photoelectric photometer, equipped with Hamamatsu R647 photomultipliers. The data were reduced with the standard quilt software package developed for the WET (Whole Earth Telescope; Nather et al. 1990). The reductions include smoothing of the sky background, and correction for extinction and linear trends. A total of 13 stars were observed, and one pulsator detected. However, due to problems with the guiding many of the sequences were rather poor, giving a $\sigma$ of about $2 \mathrm{mma}$ in the FT. Several stars showed a behaviour that could be interpreted as pulsations, but was actually due to periodic motion in the apertures. For this reason three stars were reobserved during the $\mathrm{N} 2$ run, and only the sequence with the lowest $\sigma$ is listed in Table 5.

\section{2. $C C D$ photometry}

Further NOT observations were done with the High Resolution Adaptive Camera (HIRAC) for the second run (N2 in Table 1), and the Andalucía Faint Object Spectrograph and Camera (ALFOSC) for all the remaining runs. Both HIRAC and ALFOSC were equipped with Loral, Lesser thinned, $2048 \times 2048$ CCD chips, up until January 2004, when ALFOSC was upgraded with an E2V CCD 42-40 device. The sky area available for locating reference stars was limited to $\sim 3.7 \times 3.7$ and $\sim 6.5 \times 6.5 \mathrm{arcmin}^{2}$ respectively for each of the two cameras. All CCD observations used the multi-windowed fast CCD photometry (Østensen \& Solheim 2000). Efficient fast photometry is easily achieved with this mode, since only small regions (windows) of the chip are read.

Observations were made with either a Bessell $B$-band filter (NOT \#74, Bessell 1990), for the brighter targets, and a much wider filter (NOT \#92) for the fainter ones ${ }^{4}$. This filter (hereafter referred to as the $W$, or wide filter) has the same centre as that of the $V$-filter $(5500 \AA$ ), but is a full $2750 \AA$ wide, effectively covering all bands from $B$ to $R$ with more than $90 \%$ transmission. The cycle times were mostly set to $20 \mathrm{~s}$, except for some of the brightest objects, which can have cycle times as short as $5 \mathrm{~s}$, and the faintest ones, which may have times up to $40 \mathrm{~s}$. Between 2.5 and $6.5 \mathrm{~s}$ of the cycle times are readout and shutter overheads, the exact number depending on the number of reference stars selected and the size of the readout windows.

The CCD data were reduced on-line with the Real Time Photometry (RTP) program developed by one of us as part of his Ph.D-project (Østensen 2000, see also Paper II). This software was particularly useful for the purposes of this survey, as it performs real-time reduction of the data during sequencing, displays the light-curve of the target, and computes the FT. Thus, the noise level and the presence of clear variability could be checked at the telescope, although careful data reduction was also done afterwards. The processing includes bias level

\footnotetext{
${ }^{4}$ Filter details with transmission curves for all NOT filters can be found on their web-pages; http://www. not.iac.es/.
} 
removal, flat fielding, sky subtraction, extinction correction and aperture photometry using apertures that track each star's geometrical centre.

The optimal aperture for each sequence was selected after processing all data sets with apertures of a wide range of diameters and choosing the one that gave the lowest noise in the FT. The apertures tested ranged between 10 and 40 pixels in radius, which corresponds to 1.9 and 7.6 arcsec on the sky, while the best results usually stayed within the range of 18 to 22 pixels (3.4 to 4.2 arcsec), but on nights with particularly poor seeing the optimal radius could be as high as 30 pixels $(5.7$ arcsec).

\section{Results}

A total of 309 stars deemed to lie in the predicted instability region for sdB stars were checked for variability during 25 observing runs from 1999 to 2009 . We did not apply a specific cutoff for $T_{\text {eff }}$ and $\log g$ since our estimate of these parameters were preliminary and sometimes crude. Instead we prioritised our targets by their distance from the centre of the instability region at $34 \mathrm{kK}$. In the later runs we shifted our focus more towards the region where the hybrid pulsators are located. Of all our targets, 20 were observed to pulsate with frequencies corresponding to the V361 Hya stars, which corresponds to a $\sim 6.5 \%$ ratio of fast pulsators/NOV sdBs. A further four stars were found to have such strong reflection effects that the slope was obvious even in our short light-curves. This fraction of $1.3 \%$ is only a lower limit for the $s d B+d M$ population, as there may be quite a few more objects with longer periods, which our survey for short period pulsators was not sensitive to. In fact, we know of one more reflection variable in our survey, BPS CS 22169-1, which we found to be constant in our half-hour light-curve. Since these results were not obtained at the NOT as part of this survey, they will be reported in a future paper. The 285 stars for which no obvious variability was detected are presented in Table 5.

A different detection threshold was achieved for each target, depending on its magnitude, the filter used for the observations ( $B$ or $W$ ), the length of the photometric time-series, and the weather conditions in each particular case. In the top panel of Fig. 1 the mean amplitude level in the FT $(\sigma)$ obtained for the sample is shown as function of each star's magnitude. A general trend of larger $\sigma$ for fainter objects can be distinguished, although in most cases the time-series sequence was stopped when the real-time FT showed no significant peaks in the relevant region and the highest peaks reached amplitudes $\sim 1 \mathrm{mma}$. Theoretical $\sigma$ levels as a function of magnitude for light-curves with 1, 5, 20, 100 and 300 data points are also plotted in this panel. They represent the contribution to $\sigma$ of both the scintillation and photon shot noise, and thus should be understood as the limiting accuracy in the FT obtainable from a time series with the same number of data points. The scintillation noise was computed following Young (1993), and the photon shot noise was estimated using the signal-to-noise calculator of the NOT. In both cases, a typical airmass $X=1.2$ and a typical exposure time of $20 \mathrm{~s}$ were used. Most points follow the predicted trends for light-curves with between 20 and 300 points, and the outliers are mostly due to poor weather conditions.

The middle panel of Fig. 1 shows, again as function of $\sigma$, the maximum amplitude obtained in the FT for every observed target within the $1.5-10 \mathrm{mHz}$ frequency range. The continuous line indicates the $3.7 \sigma(99 \%)$ threshold, meaning that if $A_{\max }$ is above this line, the star is considered as pulsator. The dashed line indicates the $3.0 \sigma(90 \%)$ threshold, and stars whose $A_{\max }$ falls below this line are considered as NOV, according to our

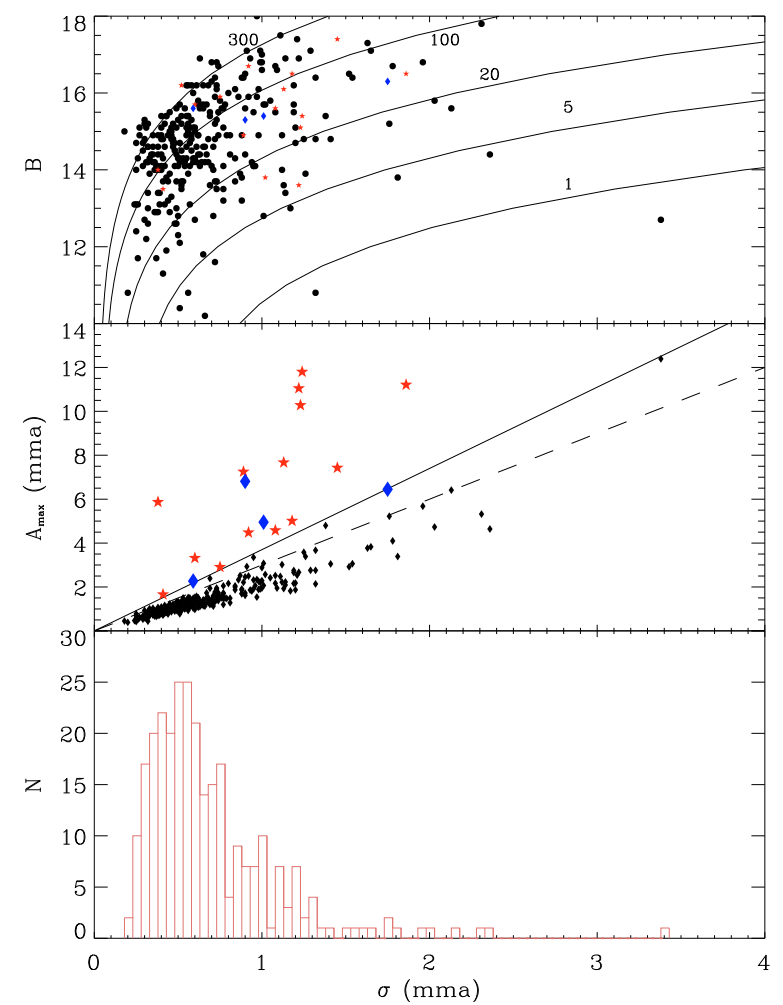

Fig. 1. Top panel: photographic $B$ magnitudes of the targets as function of $\sigma$. The labeled curves indicate the theoretical noise limits for a typical photometric observation with our system (1), and scaled to show the limit achieved for a time-series of 5, 20, 100 and 300 measurements. Middle: maximum amplitude detected in the FT for each target, as function of $\sigma$. The pulsating sdBs from this survey are marked with star symbols (published earlier) and diamonds (presented in this paper). The continuous line indicates the $3.7 \sigma$ level ( $99 \%$ confidence), and the dashed line indicates the $3.0 \sigma$ level. Bottom: Histogram of the noise level achieved for the sample.

definition. Fourteen of the twenty sdBVs discovered in this survey are marked with stars (published earlier) and four with diamonds (the new ones presented here); the remaining two pulsators, EP Psc and QQ Vir, have such large pulsation amplitudes that they lie beyond the range shown in the figure. Note that we have used only the discovery data for these plots, so the lowest amplitude pulsator, LM Dra, and the one on the $99 \%$ confidence line at $\sigma=0.75$, PG $1657+416$, have been confirmed in followup runs with higher confidence levels. The objects between the 3.0 and $3.7 \sigma$ confidence lines are discussed below, and the pulsators marked with diamonds will be discussed in Sect. 5 .

The bottom panel of Fig. 1 represents an histogram of the mean amplitude level achieved along the survey. For most of the targets, the noise level in the FT was below 1 mma (239 targets = $83 \%$ ), and in $50 \%$ of the cases a $\sigma<0.6$ mma was obtained.

\subsection{Pulsator candidates}

Ten objects in Table 5 have amplitude peaks between 3.0 and $3.7 \sigma$, higher than our NOV requirement. They must therefore still be considered pulsator candidates. Three of these have a $\sigma$ of $0.33 \mathrm{mma}$ or less, and have been ignored. The others deserve particular mention.

One object, PG $1248+164$ has such a high $\sigma$ that it is off the scale in Fig. 1. The object comes from the sample of Maxted et al. (2001) and deserves further observations. The object on 
the $99 \%$ confidence line at $\sigma=3.4$ is PG $1725+252$. Normally it would have been reobserved, but as it was realised that this object is also in the survey of Billères et al. (2002) with a comfortable $0.03 \%$ limit, no follow-up was performed. The object just below the $99 \%$ limit at $\sigma=0.7$ is KPD 2215+5037, observed during run N5. This star was also part of the survey of Billères et al. (2002) with a limit of $0.2 \%$. We note a rising trend in our light-curve on the $1 \%$ level over the 20 min covered by our observations, which could indicate that the object is a long period variable.

The three objects between the $3 \sigma$ and $3.7 \sigma$ confidence lines at $\sim 1 \sigma$ are PG $1313+132$, PG $1722+286$ and $\mathrm{J} 1351+0234$. PG 1313+132 shows variations at low frequencies, consistent with $g$-mode pulsations, which is likely considering its low temperature $\left(T_{\text {eff }}=25.6 \mathrm{kK}, \log g=5.41\right.$; Saffer et al. 1994), but uncertain as the observations were done at relatively high airmass. If we compute the noise level only for frequencies higher than $5 \mathrm{mHz}$ we get $\sigma=0.53$ and the highest peak is well below three times this value. The light-curve of PG $1722+286$ was obtained during the problematic N1 run. An attempt was made to reobserve it during $\mathrm{T} 2$, but poor weather resulted in an even worse light-curve. After a limit on pulsation of $0.08 \%$ was presented by Billères et al. (2002), no further attempts were made to observe this target. The last of the three, J1351+0234, is just noisy due to its low brightness $\left(g^{\prime}=17.1\right)$.

The three objects that lie on the $3 \sigma$ confidence line around $\sigma=2$ are HS 1813+7247, EGGR 491, and HS 2029+0301 (only the last of these is actually above the $3 \sigma$ line, but we will discuss all three). HS $1813+7247$ has a slope in the light-curve, but it is not consistent between the different reference stars, and so is most likely due to differential extinction as the $W$-band filter was used. EGGR 491 is another faint target from the SDSS sample, so the light-curve is just noisy, with no obvious features. HS 2029+0301 is also from the N1 photoelectric run, and has not been reobserved. Further observations would be required to give a more useful limit on any pulsational behaviour of this object.

\section{New pulsators}

The four pulsators found in our sample and not published elsewhere are HE 2151-1001, HS 2125+1105, PG 1033+201, and HE 1450-0957. The details of the discovery observations as well as follow-up observations on the first two done with the NOT are provided here. Table 6 includes the detailed log of observations for all these new sdBVs.

For all the pulsators we derived the frequency content of the light-curves by performing a non-linear least-squares fit to a sine function, using the highest amplitude frequency in the amplitude spectrum (top panels of Figs. 2-5). This fit was then subtracted from the light-curve to compute the residual amplitude spectrum (bottom panels). Notice that the noise level is recomputed after each prewhitening step, so that the $3.7 \sigma$ level lines in the figures drop after removing the established signal. If there was still any peak left above the significance threshold, the original light-curve was fitted to the sum of two sine functions with the established frequencies. Table 7 lists the best fitting frequencies and amplitudes, with the associated errors from the least-squares procedure.

\subsection{HE2151-1001}

This target was observed for $\sim 40$ min during the run S2. Its amplitude spectrum is shown in Fig. 2, where some signal above the
Table 6. Details from the discovery runs for the four new sdBVs.

\begin{tabular}{lcrccc}
\hline \hline Target & $\begin{array}{c}\text { Run } \\
\text { ID }\end{array}$ & \multicolumn{1}{c}{$N_{p}$} & $\begin{array}{c}\Delta t \\
\mathrm{~s}\end{array}$ & $\begin{array}{c}\sigma \\
\mathrm{mma}\end{array}$ & $\begin{array}{c}A_{\max } \\
\mathrm{mma}\end{array}$ \\
\hline HE 2151-1001 & S2 & 120 & 30 & 0.58 & 2.20 \\
& $\mathrm{~S} 3$ & 73 & 30 & 1.05 & 2.35 \\
HS 2125+1105 & $\mathrm{S} 2$ & 67 & 35 & 1.75 & 6.45 \\
& $\mathrm{ST}$ & 95 & 30 & 0.95 & 3.56 \\
& $\mathrm{~S} 3$ & 116 & 42 & 0.72 & 3.42 \\
& $\mathrm{~S} 3$ & 433 & 30 & 0.40 & 4.35 \\
& $\mathrm{~S} 3$ & 541 & 30 & 0.33 & 3.84 \\
PG 1033+201 & $\mathrm{S} 4$ & 89 & 28 & 1.17 & 4.76 \\
HE 1450-0957 & $\mathrm{T} 6$ & 118 & 20 & 1.01 & 4.95 \\
\hline
\end{tabular}

Notes. "Run ID" refers to the identifiers listed in Table $1, N_{\mathrm{p}}$ is the number of points for the specific sequence, $\Delta t$ is the sampling time interval, $\sigma$ is the mean noise level in the FT, and $A_{\max }$ is the amplitude of the highest peak.

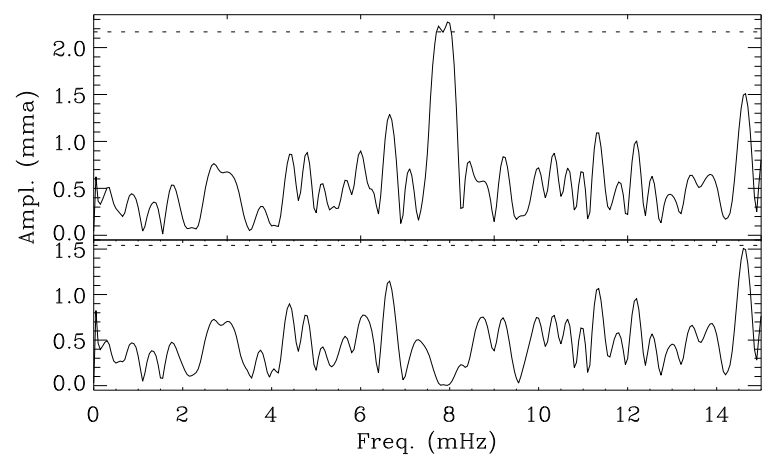

Fig. 2. Amplitude spectrum of HE2151-1001. The lower panel shows the residual spectrum after prewhitening the two frequencies listed in Table 7 from the light-curve. The dashed line indicates 3.7 times the noise level.

threshold can be distinguished at $\sim 8 \mathrm{mHz}$. This frequency range is typical for a pulsating $\mathrm{sdB}$ with these $T_{\mathrm{eff}}-\log g$ parameters (see Table 9).

Our non-linear least-squares frequency analysis revealed two low amplitude ( $3 \mathrm{mma}$ ) peaks, as listed in Table 7 . Note that the frequency distance between the two peaks $(\sim 140 \mu \mathrm{Hz})$ is within the frequency resolution $(\sim 325 \mu \mathrm{Hz})$. Another short sequence on HE 2151-1001 was obtained during S3 (see Table 6), but the noise level achieved was not sufficient to detect the oscillations above the significance threshold.

This target was observed spectroscopically as a white dwarf candidate by the SPY survey. The spectrum was analysed by Lisker et al. (2005), who found no spectral indication of a companion, but noted that it has a peculiar $\mathrm{H} \alpha$ profile. The 2MASS photometry $(J=16.53, H=16.37, K=15.91)$ indicates a main sequence companion, but this is uncertain as the values are close to the faint limit of the 2MASs survey. The NOMAD catalogue (Naval Observatory Merged Astrometric Dataset, Zacharias et al. 2005) provides reasonably good photographic magnitudes $(B=15.25, V=15.57, R=16.09)$, and the $U V$ magnitudes $(F U V=14.55, N U V=14.87)$ from the GALEX satellite (Martin et al. 2005) all indicate colours typical for a hot subdwarf.

\subsection{HS $2125+1105$}

The faint $(B=16.3)$ HS $2125+1105$ was first observed during run S2. A $6.4 \mathrm{mma}$ peak was detected at $6.89 \mathrm{mHz}$, although 


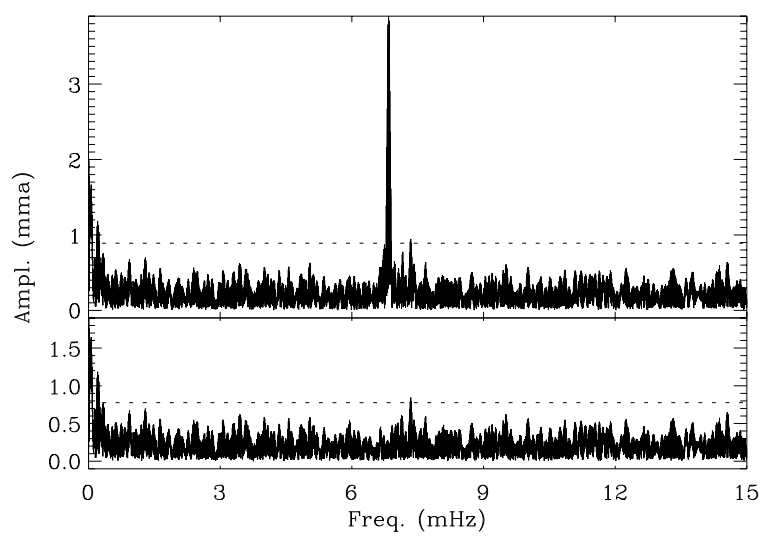

Fig. 3. Amplitude spectrum of HS $2125+1105$. The lower panel shows residual spectrum after subtracting one frequency from the original light-curve. The dashed line indicates 3.7 times the noise level. The peak at $7.3 \mathrm{mHz}$ is just above the $3.7 \sigma$ level if the low frequency domain is excluded when estimating $\sigma$.

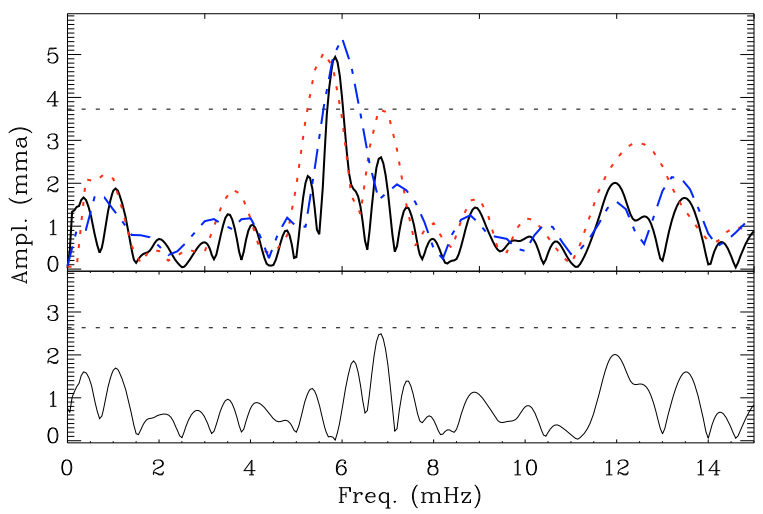

Fig. 4. Amplitude spectrum of PG 1033+201. The lower panel shows the residual spectrum after prewhitening the two frequencies listed in Table 7 from the light-curve. We have also plotted the amplitude spectrum of the first half (red dashed curve) and second half (blue short dash, long dash curve), to demonstrate that the main peak is present with the same amplitude in both halves of the dataset. The dashed line indicates 3.7 times the noise level.

its significance level was exactly 3.7 times the noise level (see Table 6). A year later, a slightly longer light-curve with better photometric conditions was obtained during run ST, which reduced the noise level to $0.95 \mathrm{mma}$. However, a peak detected at the same frequency also dropped its amplitude to $3.6 \mathrm{mma}$, again at exactly the 3.7 times the noise level. During run S3, the object was observed on each of the three nights as indicated in Table 6, and the good conditions finally allowed us to get reliable confirmation of the main peak. The amplitude spectrum obtained from the combined light-curve is shown in the upper panel of Fig. 3. In Table 7 the results of a frequency analysis are listed. A $4 \mathrm{mma}$ peak at $6.83 \mathrm{mHz}$ is detected, but this time at a reassuringly high $S / N$. In this sequence a second peak at $7.34 \mathrm{mHz}$ was also detected, as listed in Table 7. The bottom panel of Fig. 3 shows the residual amplitude spectrum after prewhitening the main frequency from the light-curve.

In order to check the stability of this mode, an additional short sequence was taken in $S 4$. The same $f_{1}$ was found, this time with a 4.7 mma amplitude.

This HS star was not part of the original sample of sdB stars from Edelmann (2003), but was observed as part of the SPY survey and was analysed by Lisker et al. (2005). The star has reliable optical photometry (but no spectrum) in the SDSS

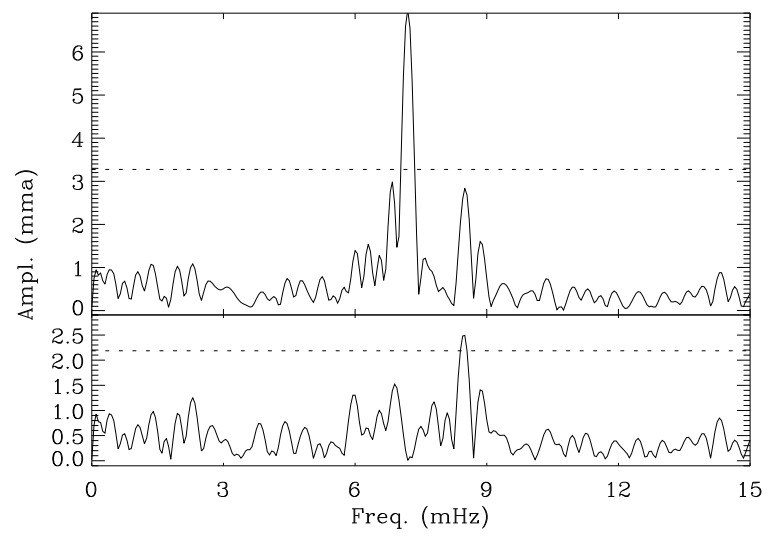

Fig. 5. Amplitude spectrum of HE 1450-0957 (top) and residual amplitude spectrum after subtracting the main peak (bottom), with $3.7 \sigma$ noise levels (dashed lines).

Table 7. Pulsation properties for the four new pulsators.

\begin{tabular}{lcccr}
\hline \hline Name & Id & $\begin{array}{c}\text { Frequency } \\
{[\mu \mathrm{Hz}]}\end{array}$ & $\begin{array}{c}\text { Amplitude } \\
{[\mathrm{mma}]}\end{array}$ & $S / N$ \\
\hline HE 2151-1001 & $f_{1}$ & $7931 \pm 63$ & $3.04 \pm 1.81$ & 6.5 \\
& $f_{2}$ & $7791 \pm 64$ & $3.00 \pm 1.80$ & 6.5 \\
HS 2125+1105 & $f_{1}$ & $6835.66 \pm 0.07$ & $4.05 \pm 0.19$ & 19.6 \\
& $f_{2}$ & $7342.35 \pm 0.35$ & $0.85 \pm 0.19$ & 4.1 \\
PG 1033+201 & $f_{1}$ & $5850 \pm 27$ & $4.9 \pm 0.6$ & 8.1 \\
& $f_{2}$ & $6837 \pm 53$ & $2.5 \pm 0.6$ & 4.5 \\
HE 1450-0957 & $f_{1}$ & $7202 \pm 8$ & $6.9 \pm 0.4$ & 13.7 \\
& $f_{2}$ & $8475 \pm 21$ & $2.5 \pm 0.4$ & 5.2 \\
\hline
\end{tabular}

Notes. Pulsation frequencies and amplitudes are given as derived by non-linear least-square fitting to the light-curves. The $S / N$ is computed after prewhitening the detected frequencies from the signal.

survey $\left(u^{\prime}=16.13, g^{\prime}=16.36, r^{\prime}=16.78, i^{\prime}=17.09, z^{\prime}=17.33\right)$. There is no indication of a main sequence companion from the spectroscopy or SDSS photometry. The object is well below the faint limit of the 2MASS survey, so no IR colours are available. The GALEX photometry is FUV $=15.6, \mathrm{NUV}=15.8$.

\section{3. $P G 1033+201$}

PG 1033+201 was one among 15 targets observed with the $B$-band filter during the technical night T7. During the run we had some problems with the observing system, which resulted in some frames in the photometric sequence being dropped. For this reason the real-time processing program was unable to process the entire sequence. Due to these problems we stayed on target almost 40 min, which is considerably longer than our standard sequence on such bright targets. Eventually, we terminated the observations in order to reinitialise the CCD system, but we never returned to reobserve this star. During the careful reprocessing of the photometry for this paper, we excluded two lost and one bad data point from the 118 frame sequence, and performed a regular frequency analysis of the cleaned light-curve. The amplitude spectrum is shown in the top panel of Fig. 4 as a continuous line. A clear peak at $5.8 \mathrm{mHz}$ is well above the $3.7 \sigma$ (horizontal dashed) line. We divided the light-curve into two equal halves, and we can see from the corresponding spectra (also shown in Fig. 4 as red dotted and blue dash-dotted curves), that the main period is present in both halves with the same amplitude. A second frequency at $6.8 \mathrm{mHz}$ is significant only in the first part, indicating that it is either spurious or a combination of 
frequencies suffering cancellation from beating within our short sequences. This exercise demonstrates how short-period pulsations can be detected with reasonable confidence even in short $(\sim 20 \mathrm{~m})$ light-curves, as long as one acquires adequate signalto-noise in the individual data-points. The bottom panel displays the residual amplitude spectrum after subtracting the frequency at $5.8 \mathrm{mHz}$. The remaining peak at $\sim 6.8 \mathrm{mHz}$ becomes significant when the noise is computed in the prewhitened spectrum (see Table 7). We conclude that PG 1033+201 appears to be a typical short-period sdB pulsator with a main pulsation amplitude of $5 \mathrm{mma}$, with likely further frequencies at the $2 \mathrm{mma}$ level.

This object was included in our sample based on the decomposition by Allard et al. (1994), who estimated the system to be an $\mathrm{sdB}+\mathrm{F} 9$ composite from their Cousins $B V R I$ photometry $(V=15.67, B-V=-0.17, V-R=-0.01, R-I=+0.06)$, and provided a colour temperature for the primary of $31.5 \mathrm{kK}$. The object occurs in the SDSS survey as J103638.93+195202.2, and has photometry $\left(u^{\prime}=15.14, g^{\prime}=15.39, r^{\prime}=16.80, i^{\prime}=16.01\right.$, $\left.z^{\prime}=16.21\right)$ but no spectroscopy, which prevents us from acquiring a better temperature and a gravity estimate. The 2MASS IR photometry clearly supports the notion of a main-sequence companion $(J=15.46, H=15.16, K=15.13$, i.e. $J-H>+0.3$, see Reed \& Stiening 2004).

\subsection{HE 1450-0957}

HE 1450-0957 was one of the three targets surveyed during the final run of the programme (S6). A rather long light-curve was obtained (1h5min), since its oscillations were already clear as the data were processed as they arrived. Its amplitude spectrum (upper panel of Fig. 5) reveals the multimode behaviour of this target. At almost $7 \mathrm{mma}$, the main peak at $7.2 \mathrm{mHz}$ has the highest amplitude of the four new pulsators presented here. A significant second peak is also detected in the amplitude spectrum of HE 1450-0957 (see bottom panel of Fig. 5), but more low amplitude modes are likely to remain in the range $6-9 \mathrm{mHz}$. The frequencies derived from this discovery light-curve are also listed in Table 7.

This object came into our sample from the HE stars surveyed by SPY (Lisker et al. 2005). It is included in the EdinburghCape survey as EC 14507-0957 where reliable photometry can be found $(V=15.27, U-B=-1.04, B-V=-0.21)$. As noted by Reed \& Stiening (2004), its 2MASS magnitudes $(J=15.58$, $H=15.41, K=15.36)$ are indicative of a main sequence companion, but this companion must be very weak in the optical as Lisker et al. (2005) do not detect any trace of it in their VLT/UVES spectrum.

\section{Summary and discussion}

The NOT search programme for pulsating sdB stars has been a great success, contributing twenty new pulsators to the known population of short period sdBVs. Together with the two new pulsators presented by Kilkenny et al. (2009), another two recently found by Barlow et al. (2009, 2010), and HE 0230-43235 , our latest tally brings the total up to 49 . Our effort to implement an efficient system for CCD photometry at the NOT has certainly been fruitful, producing on average one new pulsator for every three nights of observations. This is a substantially higher efficiency than any other group has reported.

\footnotetext{
5 Originally reported as "an unusual hot subdwarf pulsator" by Koen (2007) but now confirmed to be a more regular sdBV by Kilkenny (priv. comm.).
}

\subsection{Follow-up}

Many of our pulsators have already received detailed follow-up. Most effort has been put in on V391 Peg, which was the subject of several campaigns, and its pulsation periods are still being monitored regularly (Silvotti et al. 2007). The original aim of this monitoring was to detect evolutionary period changes, but the periodic variation that was found is more likely to be caused by an orbiting planet inducing period variations through the light travel time effect. Several other stars in our sample are now regularly monitored for variations in the pulsation period under the EXOTIME project (Lutz et al. 2009). As a substantial time base of these observations develops, we expect to detect more planethosting hot subdwarf stars.

QQ Vir was the first sdBV for which spectroscopic lineprofile variations were used to constrain its main pulsation mode (Telting \& Østensen 2004), and was the subject of an extensive photometric campaign in 2003 (Silvotti et al. 2006) revealing 15 pulsation frequencies. The detailed frequency spectrum from this campaign allowed Charpinet et al. (2006) to obtain an asteroseismic solution using the forward method. QQ Vir also became the first star from our sample to be targeted with highresolution spectroscopy with the VLT in 2008 (Telting et al. 2010).

LM Dra was followed extensively by Reed et al. (2007a) with multisite observations between 2003 and 2004, and in spite of its extremely low pulsation amplitudes they detected six pulsation periods with amplitudes between 1.0 and $2.2 \mathrm{mma}$. V429 And and V1636 Ori were followed by Reed et al. (2007b) in Nov.-Dec. 2005, revealing a rich frequency spectrum with fourteen significant peaks between 0.45 and $4.35 \mathrm{mma}$ in V429 And, and a simpler spectrum with only three significant peaks between 0.8 and $11.1 \mathrm{mma}$ in V1636 Ori. Reed et al. (2007b) also report that LS Dra appears to be monoperiodic, but with a highly variable amplitude, changing from a maximum of 5.26 to a minimum of 0.87 mma over their campaign spanning 47 days. They further confirm three of the four pulsation periods in V387 Peg.

J1717+5805 was targeted with high-speed simultaneous 3-channel photometry using ULTRACAM on the WHT, as reported by Aerts et al. (2006), resolving the broad peak described in Paper V into two separate frequencies.

\subsection{Summary}

The four new pulsators presented in this paper certainly deserve more attention as well. Confirming the pulsations in PG 1033+201 would be a priority, but an intensive campaign would probably be required to disentangle its multiperiodic nature. The pulsations observed in HE 2151-1001 are barely above our significance threshold and need to be confirmed. HS $2125+1105$ is clearly a stable pulsator, as the same oscillation period was detected in six sequences spanning four years, but we would suspect more frequencies to be discovered with more dedicated follow-up. HE 1450-0957 is also a convincing case where residual power in the amplitude spectrum between 6 and $10 \mathrm{mHz}$ indicates the presence of unresolved pulsations that could be revealed with dedicated follow-up. The existence of pulsations in this star was recently confirmed by observations from South Africa (C. Koen, priv. comm.).

In Table 8 we compare the fraction of pulsators detected in each of the different samples we have drawn our candidates from. A fraction of pulsators between 5 and $12 \%$ is clear when targets are selected based on spectroscopic temperature 
Table 8. Comparison with the results of other surveys for pulsating sdBs (upper part) and our own survey divided into subsamples (lower part).

\begin{tabular}{lrrrrl}
\hline \hline Sample & Size & \multicolumn{2}{c}{ Pulsators } & Fract. & Preselection \\
& $\#$ & $\mathrm{~S}$ & $\mathrm{C}$ & $\%$ & on $T_{\text {eff }}$ \\
\hline South Africa & $\sim 1200$ & 13 & 7 & 1.7 & No \\
Billeres & 74 & 0 & 4 & 5.4 & Yes \\
Dreizler & 12 & 1 & 0 & 8 & Yes \\
HS & 80 & 9 & 0 & 10 & Yes, -comp. \\
HE & 23 & 0 & 0 & 0 & Yes \\
SPY & 27 & 3 & 0 & 11 & Yes \\
SDSS & 40 & 0 & 5 & 12.5 & Yes \\
BG & 11 & 1 & 0 & 9 & Yes \\
Saffer & 24 & 1 & 0 & 4 & Yes \\
Maxted & 24 & 0 & 0 & 0 & Yes \\
colour & $\sim 50$ & 0 & 2 & 4 & Poor \\
\hline
\end{tabular}

Notes. "Size" indicates the number of targets checked for variability in each sample. The number of pulsators discovered are separated into apparently single (S) and spectroscopic composites (C), but note that binaries with invisible (WD or dM) companions are included in the column for single pulsators. We also specify if the sample was preselected to contain only subdwarfs in the instability region or not. Note that a few of the pulsators are present in more than one sample, and that we keep the HS and HE stars observed only as part of the SPY survey apart from the HS and HE samples.

estimates. We estimate that it should be possible to obtain a pulsation fraction of about $10 \%$, when starting with an undepleted sample and reaching a noise level below $1 \mathrm{mma}$. This could probably be increased by focusing only on the hot end of the EHB, between 31 and $36 \mathrm{kK}$, at the cost of sacrificing discoveries of the rarer pulsators at the cool end of the instability region.

That the colour selected sample has a lower success fraction than the samples based on spectroscopy is not surprising, but it is surprising that there are so few pulsators in the sample from Saffer et al. (1994). The total number of sdB stars in that sample hotter than $28 \mathrm{kK}$ are 44 , of which we observed 16 (plus another eight that were outside the borders of the instability region). Excluding the known $g$-mode pulsators on the cool end of that sample, we have two short period sdBVs in 41 stars; QQ Vir found by us and V1078 Her found by Bonanno et al. (2003). A large number of stars from this sample were surveyed by Billères et al. (2002), and the remaining stars have been checked by other observers (but no limits have been published), so we do not expect more clear pulsators in this sample, although some low amplitude variables may have been missed. Similarly, the number of sdB stars in the instability region in the sample of Maxted et al. (2001) is 34. We found no pulsators in this sample, but it has been heavily exploited by other groups; Billères et al. (2002) surveyed 14 of them and found one: UY Sex. When combining information from this and other unpublished surveys, we can only find a single star that has yet to be surveyed. Thus, the best fraction from the Maxted et al. (2001) sample we can infer is $3 \%$, which is lower than what we have found in the HS, SPY and SDSS samples, but with such small samples this is barely significant.

\subsection{The big picture}

We have compiled all the known short period sdB pulsators in Table 9, using data from the literature and added the results from this paper. We included preliminary physical parameters on two new pulsators published by Kilkenny et al. (2009), based on recent low-resolution spectroscopy from NOT/ALFOSC (3250-6150 $\AA$ at $\sim 4.5 \AA$ resolution), using the same atmosphere models as in our other determinations. A few stars still lack temperature and gravity estimates from spectroscopy, but we have supplied values from our own ALFOSC spectroscopy where available. The table may also not be entirely complete. PG $0856+121$, for which Piccioni et al. (2000) reported what they termed "a pulsation episode", was dropped from the table as repeated attempts (Reed, priv. comm) have failed to confirm any pulsations. Such brief pulsation episodes may be common in sdB stars, but they are hard to catch and characterise.

The first sdB pulsator candidate in a globular cluster was reported by Randall et al. (2009a), but while the single clear period at $114 \mathrm{~s}$ makes this star a very likely sdBV, spectroscopic confirmation remains to be done. The same is the case for the pulsator candidate reported by Silvotti et al. (2009), which was found in the Kepler satellite field-of-view, with a likely pulsation period of $125 \mathrm{~s}$ found at the $3.5 \sigma$ level.

We note that the number of pulsators in Table 9 with $\mathrm{F}-\mathrm{K}$ companions are 18 of $49=37 \%$, which is compatible with the fraction of spectroscopic composites we found in the SDSS as a whole. Pulsators also occur in systems with close white dwarf or M-dwarf companions, which means that pulsations appear independently of whether the subdwarf was formed through stable Roche-lobe overflow or via common envelope ejection. The stability of the pulsations for stars such as V391 Peg has revealed that some sdBs are definitely single, which implies that a formation channel must exist that produces single sdB stars that are otherwise indistinguishable from those produced via the channels that produces wide $\mathrm{sdB}+\mathrm{F}-\mathrm{K}$ or short period binaries.

In Fig. 6a we show the location in the $\left(T_{\text {eff }}, \log g\right)$ plane of the pulsators from our sample (blue) together with pulsators from the literature (red), as listed in Table 9, with symbol size proportional to $A_{\max }$. As our sample stars are fairly evenly spaced along the canonical EHB strip, it is immediately clear that pulsations are more common on the hot side than on the cool side, but the amplitudes are often much higher in the cool, low gravity region. The figure does not really reproduce the gap around $T_{\text {eff }} \sim 30 \mathrm{kK}$ between the main group of pulsators on the hot end of the EHB and the cool group of $g$-mode and hybrid pulsators seen in Fig. 3 of Østensen (2009), where the spectroscopy was obtained from the independent BG survey (Green et al. 2008). This may be due to the fact that diverse methods and model grids are used in the temperature determinations collected from the literature, and this can produce scatter as high as $2 \mathrm{kK}$ in $T_{\text {eff }}$ and $0.2 \mathrm{dex}$ in $\log g$. If we plot only the stars for which we ourselves have obtained the fits on a consistent model grid, the gap is indeed present.

The main cluster of pulsators correlates very well with the initial instability strip predicted by Charpinet et al. (1997), with only a few rare pulsators appearing to be anomalous. While the sample presented here contains no stars hotter than $39 \mathrm{kK}$, we have observed quite a few such stars without finding any with significant variability. But these are classified as sdO or He-sdO stars, and will be presented in a future paper. Candidates in that sample were selected from very diverse surveys and ended up having a wide range of physical parameters, as stars matching the properties of the only known sdO pulsator, J16007+0748 (Woudt et al. 2006) are extremely rare, preventing an effective preselection such as we made for the sdB sample.

Figure $6 \mathrm{~b}$ shows the surface gravities plotted against the range of pulsation periods listed in Table 9. As the $\log g$ values are derived with many different methods, the systematic errors can be substantially larger than \pm 0.1 dex. Still, the expected relationship between pulsation periods and surface gravity seems 
R. H. Østensen et al.: A survey for pulsating sdB stars

Table 9. All short period sdBV stars published to date.

\begin{tabular}{|c|c|c|c|c|c|c|c|c|c|c|}
\hline Name & Survey name & $\begin{array}{l}m_{V} \\
\mathrm{mag}\end{array}$ & $\begin{array}{l}T_{\text {eff }} \\
\mathrm{kK}\end{array}$ & $\begin{array}{c}\log g \\
\operatorname{dex}\end{array}$ & $\begin{array}{c}\log y \\
\operatorname{dex}\end{array}$ & $C$ & $\begin{array}{r}P_{\text {range }} \\
\mathrm{s}\end{array}$ & $\begin{array}{l}A_{\max } \\
\mathrm{mma}\end{array}$ & $N_{\mathrm{P}}$ & References \\
\hline V361 Hya & EC 14026-2647 & 15.3 & 34.7 & 6.10 & $\ldots$ & G2 & $134-144$ & 12 & 2 & 1,4 \\
\hline EOCet & PB 8783 & 12.3 & 35.7 & 5.70 & $\ldots$ & F0 & $94-136$ & 9 & 7 & 2,4 \\
\hline UX Sex & EC 10228-0905 & 15.9 & 33.5 & 6.00 & $\ldots$ & G2 & $140-152$ & 14 & 3 & 3,4 \\
\hline V4640 Sgr & EC 20117-4014 & 12.5 & 34.8 & 5.87 & $\ldots$ & F5 & $137-158$ & 4 & 3 & 4 \\
\hline UY Sex & PG 1047+003 & 13.5 & 33.2 & 5.80 & -2.00 & $a, b$ & 104-175 & 10 & 18 & $5, k 1$ \\
\hline NY Vir & PG 1336-018 & 13.5 & 31.3 & 5.60 & -2.93 & M5 & $97-205$ & 10 & 28 & $6, k 2, v 2$ \\
\hline V2203 Cyg & KPD 2109+4401 & 13.4 & 31.8 & 5.79 & -2.23 & $a$ & $182-213$ & 9 & 8 & $7, z 1, h 1$ \\
\hline V338 Ser & PG 1605+072 & 12.8 & 32.3 & 5.25 & -2.53 & $a$ & $350-573$ & 64 & 50 & 8,12 \\
\hline KL UMa & Feige 48 & 13.5 & 29.5 & 5.50 & -2.93 & WD & $343-379$ & 6 & 4 & $9, h 1, o 2$ \\
\hline DT Lyn & PG 0911+456 & 14.6 & 31.9 & 5.77 & -2.55 & a & 149-192 & 7 & 7 & $10, r 5$ \\
\hline KY UMa & PG $1219+534$ & 13.2 & 34.3 & 5.95 & -1.50 & $a, b$ & $128-149$ & 9 & 4 & $10, h 1$ \\
\hline AQ Col & EC 05217-3914 & 15.6 & 31.3 & 5.76 & -3.00 & $\ldots$ & $215-218$ & 8 & 8 & $11, r 3$ \\
\hline V1405 Ori & KUV 04421+1416 & 15.1 & 32.0 & 5.72 & -2.50 & $\mathrm{dM}$ & $185-232$ & 20 & 7 & $11, r 4$ \\
\hline V2214 Cyg & KPD 1930+2752 & 13.8 & 35.2 & 5.61 & -1.50 & WD & $145-332$ & 5 & 44 & $13, g 1$ \\
\hline LM Dra & PG 1618+563B & 13.5 & 33.9 & 5.80 & -1.60 & $\mathrm{~F}^{c}{ }^{c}$ & $108-144$ & 6 & 2 & $14, r 3$ \\
\hline DV Lyn & HS 0815+4243 & 16.1 & 33.7 & 5.95 & -2.10 & $\ldots$ & $126-131$ & 7 & 2 & 15 \\
\hline V384 Peg & HS $2149+0847$ & 16.5 & 35.6 & 5.90 & -1.80 & $\ldots$ & $142-159$ & 11 & 5 & 15 \\
\hline V391 Peg & HS 2201+2610 & 13.6 & 29.3 & 5.40 & -3.00 & $\mathrm{Pl}$ & $347-351$ & 10 & 3 & $15, s 1$ \\
\hline LS Dra & HS $1824+5745$ & 15.6 & 33.1 & 6.00 & -1.52 & $\ldots$ & 139 & 5 & 1 & $16, r 1$ \\
\hline V387 Peg & HS $2151+0857$ & 16.5 & 34.5 & 6.10 & -1.37 & $\mathrm{~F}-\mathrm{K}$ & $129-151$ & 4 & 5 & $16, r 1$ \\
\hline V429 And & HS 0039+4302 & 15.1 & 32.4 & 5.70 & -2.20 & $a$ & $182-235$ & 8 & 4 & 16 \\
\hline V1636 Ori & HS 0444+0458 & 15.4 & 33.8 & 5.60 & -1.85 & $a$ & $137-168$ & 13 & 2 & 16 \\
\hline EK Psc & PG 0014+067 & 16.3 & 34.1 & 5.77 & -1.68 & $b$ & 137-169 & 3 & 13 & $17, c 1, v 1$ \\
\hline EP Psc & PG 2303+019 & 16.2 & 35.3 & 5.74 & -1.70 & MS? & $128-145$ & 16 & 3 & 18 \\
\hline QQ Vir & PG $1325+101$ & 13.8 & 34.8 & 5.81 & -1.65 & $a, b$ & 94-168 & 26 & 14 & $18, t 1, s 2$ \\
\hline$D W L y n$ & HS $0702+6043$ & 14.7 & 28.4 & 5.35 & -2.70 & $a$ & $363-384$ & 22 & 2 & 19,53 \\
\hline V1078 Her & PG $1613+426$ & 14.1 & 34.4 & 5.97 & -1.65 & $a$ & 144 & 5 & 1 & 20 \\
\hline PG 0048+091 & $\ldots$ & 14.3 & 34.2 & 5.69 & -3.00 & F5 & $90-192$ & 6 & 28 & $21, r 3, \sharp$ \\
\hline PG 0154+182 & $\ldots$ & 15.3 & 35.8 & 5.80 & -1.67 & G-K & $110-164$ & 10 & 6 & $21, r 1, \not$ \\
\hline $\mathbf{J} 1717+5805$ & $\ldots$ & 16.7 & 34.4 & 5.75 & -1.80 & G9 & $137-144$ & 6 & 2 & $22, \dagger$ \\
\hline Balloon 090100001 & $\ldots$ & 11.8 & 29.4 & 5.33 & -2.54 & $a, b$ & $178-356$ & 60 & 3 & $23, o 1$ \\
\hline PG 1419+081 & $\ldots$ & 14.9 & 33.3 & 5.85 & -1.80 & G5 & 143 & 7 & 1 & $24, \dagger$ \\
\hline J1445+0002 & $\ldots$ & 17.4 & 35.9 & 5.75 & -1.60 & G0 & $120-142$ & 8 & 3 & $24, \uparrow$ \\
\hline $\mathrm{J} 1642+4252$ & $\ldots$ & 15.7 & 32.4 & 5.80 & -2.00 & G0 & $130-138$ & 3 & 2 & $24, \dagger$ \\
\hline EC 09582-1137 & PG 0958-116 & 15.2 & 34.8 & 5.79 & -1.68 & $a$ & 136-169 & 8 & 5 & $25, r 6$ \\
\hline EC 11583-2708 & $\ldots$ & 14.4 & $\ldots$ & $\ldots$ & $\ldots$ & MS & 114-149 & 3 & 4 & 25 \\
\hline EC 20338-1925 & BPS CS 22880-18 & 15.6 & 35.5 & 5.75 & -1.71 & $a$ & $135-168$ & 27 & 5 & 25, \\
\hline RAT J0455+1305 & $\ldots$ & 17.2 & 29.2 & 5.20 & $\ldots$ & $\ldots$ & $363-374$ & 19 & 2 & $26, b 1$ \\
\hline PG 1657+416 & $\ldots$ & 15.9 & 32.2 & 5.80 & -1.60 & G5 & $125-143$ & 4 & 5 & $27, \dagger$ \\
\hline 2M0415+0154 & $\ldots$ & 14.0 & 34.0 & 5.80 & -1.60 & $a, b$ & $144-149$ & 6 & 3 & 28 \\
\hline EC 01541-1409 & $\ldots$ & 12.2 & 37.1 & 5.71 & -1.21 & $a$ & $78-146$ & 10 & 6 & 29 , \\
\hline EC 22221-3152 & $\ldots$ & 13.4 & 35.1 & 5.85 & -1.47 & $a$ & $84-176$ & 9 & 10 & 29 , \\
\hline JL 166 & $\ldots$ & 15.0 & 34.4 & 5.75 & -0.80 & MS & $97-167$ & 4 & 10 & 30 \\
\hline CS 1246 & CS $124636.2-631549$ & 14.6 & 28.5 & 5.46 & -2.00 & $b$ & 372 & 35 & 1 & 31 \\
\hline HE 0230-4323 & $\ldots$ & 13.8 & 31.6 & 5.60 & -2.58 & $\mathrm{dM}$ & $282-310$ & 8 & 3 & $32, l 1$ \\
\hline HE 2151-1001 & $\ldots$ & 15.6 & 35.0 & 5.70 & -1.60 & MS & $126-128$ & 3 & 2 & $\dagger, l 1$ \\
\hline HS 2125+1105 & $\ldots$ & 16.3 & 32.5 & 5.76 & -1.86 & $a$ & $136-146$ & 4 & 1 & $\dagger, l 1$ \\
\hline PG 1033+201 & $\ldots$ & 15.4 & 31.5 & $\ldots$ & $\ldots$ & F9 & 146-171 & 5 & 2 & $\dagger, a 1$ \\
\hline HE 1450-0957 & EC $14507-0957$ & 15.3 & 34.6 & 5.79 & -1.29 & MS & $118-139$ & 7 & 3 & $\dagger, l 1$ \\
\hline
\end{tabular}

Notes. Pulsators discovered by our survey are listed with their names in bold face, and hybrid DW Lyn type pulsators in italics. $C$ gives the class of the companion, where available, and the notes in that column mark: ${ }^{(a)}$ no IR excess from 2MASS or SDSS photometry; ${ }^{(b)}$ no detectable RV variations on a time-scale of hours or days; ${ }^{(c)}$ the F3 companion to LM Dra is separated by 3.7", but it is unclear whether the subdwarf itself is single. $P_{\text {range }}$ gives the range of periods observed, excluding marginal detections, harmonics and $g$-modes, $A_{\max }$ gives the highest amplitude reported, and $N_{\mathrm{P}}$ the number of independent periods reported.

References. Discovery papers: ${ }^{1}$ Kilkenny et al. (1997); ${ }^{2}$ Koen et al. (1997); ${ }^{3}$ Stobie et al. (1997a); ${ }^{4}$ O’Donoghue et al. (1997); ${ }^{5}$ Billeres et al. (1997); O’Donoghue et al. (1998); ${ }^{6}$ Kilkenny et al. (1998); ${ }^{7}$ Billeres et al. (1998); Koen (1998); ${ }^{8}$ Koen et al. (1998a); ${ }^{9}$ Koen et al. (1998b); ${ }^{10}$ Koen et al. (1999b); ${ }^{11}$ Koen et al. (1999a); ${ }^{12}$ Kilkenny et al. (1999); ${ }^{13}$ Billéres et al. (2000); ${ }^{14}$ Paper I; ${ }^{15}$ Paper II; ${ }^{16}$ Paper III; ${ }^{17}$ Brassard et al. (2001); ${ }^{18}$ Paper IV; ${ }^{19}$ Dreizler et al. (2002); ${ }^{20}$ Bonanno et al. (2003); ${ }^{21}$ Koen et al. (2004); ${ }^{22}$ Paper V; Aerts et al. (2006); ${ }^{23}$ Oreiro et al. (2004); ${ }^{24}$ Paper VI; ${ }^{25}$ Kilkenny et al. (2006); ${ }^{26}$ Ramsay et al. (2006); ${ }^{27}$ Paper VII; ${ }^{28}$ Paper VIII; ${ }^{29}$ Kilkenny et al. (2009); ${ }^{30}$ Barlow et al. (2009); ${ }^{31}$ Barlow et al. (2010); ${ }^{32}$ Koen (2007); Kilkenny (priv.comm.); ${ }^{\dagger}$ this paper. Supplementary data: ${ }^{a 1}$ Allard et al. (1994); ${ }^{b 1}$ Baran \& Fox Machado (2010); ${ }^{c 1}$ Charpinet et al. (2005); ${ }^{g 1}$ Geier et al. (2007); ${ }^{h 1}$ Heber et al. (2000); ${ }^{k 1}$ Kilkenny et al. (2002); ${ }^{22}$ Kilkenny et al. (2003); ${ }^{l 1}$ Lisker et al. (2005); ${ }^{o 1}$ Oreiro et al. (2005); ${ }^{o 2}$ O'Toole et al. (2004); ${ }^{r 1}$ Reed et al. (2006); ${ }^{r 2}$ Reed et al. (2007a); ${ }^{r 3}$ Reed et al. (2007b); ${ }^{r 4}$ Reed et al. (2010); ${ }^{r 5}$ Randall et al. (2007); ${ }^{r 6}$ Randall et al. (2009b); ${ }^{s 1}$ Silvotti et al. (2002a, 2007); ${ }^{s 2}$ Silvotti et al. (2006); ${ }^{s 3}$ Schuh et al. (2006); ${ }^{t 1}$ Telting \& Østensen (2004); ${ }^{v 1}$ Vučković et al. (2006); ${ }^{v 2}$ Vučković et al. (2007); ${ }^{z 1}$ Zhou et al. (2006); ${ }^{\ddagger}$ Our determination of $T_{\text {eff }}, \log g$ and $\log y$. 

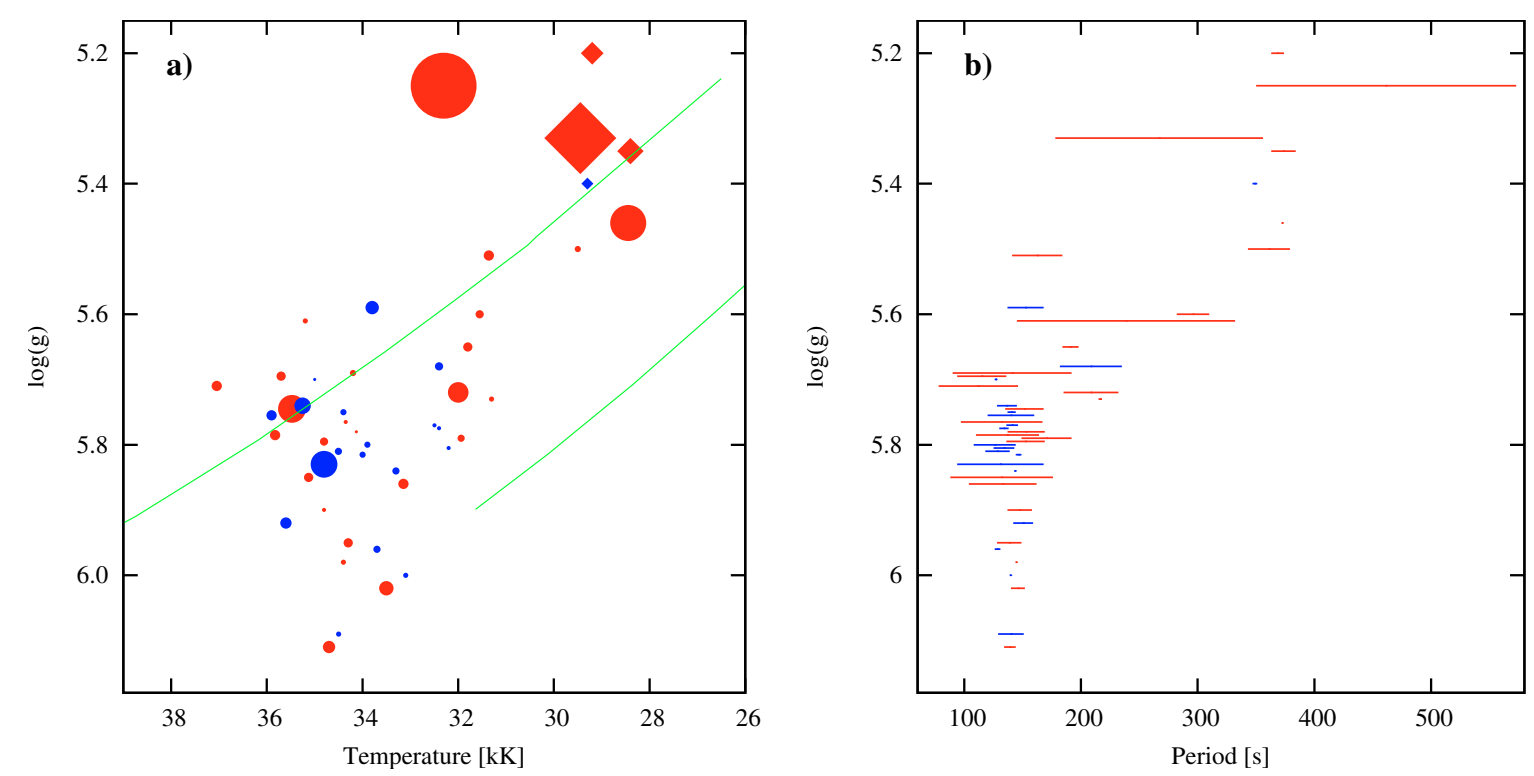

Fig. 6. a) $T_{\text {eff }}-\log g$ diagram for the objects in Table 9 . Filled circles indicate V361 Hya type pulsators and diamonds indicate hybrid DW Lyn type pulsators. The size of the symbols is proportional to the pulsation amplitude, $A_{\max }$. The nineteen sdBVs from this survey that we have $T_{\text {eff }}$ and $\log g$ for are shown in blue, and short period pulsators from other surveys in red. The green lines indicate the canonical zero age EHB (lower line) and terminal age EHB for an $0.47 \mathrm{M}_{\odot}$ core (from Kawaler \& Hostler 2005) with envelope mass increasing from bottom to top. The ZAEHB starts at a $\log g$ of 5.9 with an envelope mass fraction of only $0.02 \%$, and the objects that appear to lie on the extension of this line cannot be explained by the canonical models. b) $P-\log g$ diagram for the same pulsators. The bars indicate the range of detected pulsation periods in any particular star, excluding harmonics and $g$-modes. The $\log g$ values have been shifted by up to \pm 0.04 dex to avoid overlaps.

to hold up quite well (see Koen et al. 1999b). The relationship appears to break down for objects with surface gravities higher than 5.8 dex, but this may not be a real effect. Evolutionary tracks for EHB stars with any envelope thick enough to sustain pulsations can hardly reach higher surface gravities than about 5.9 dex (note the lower end of the canonical ZAEHB in Fig. 6). On the other hand, it is well known that the very high rotation rates in binary systems, high metallicity, as well as the pulsations themselves, give rise to broadening of atmospheric lines. If these broadening effects are not accounted for in the models used for estimating the atmospheric parameters, the fitting procedure may converge towards a too high gravity to compensate.

\section{Conclusions and outlook}

The interest in $\mathrm{sdB}$ pulsators can only increase in the future, when satellite missions such as Kepler (Christensen-Dalsgaard et al. 2007) start to find numerous new sdBVs and observe them with unprecedented frequency resolution and at lower pulsation amplitudes than have ever been achieved from the ground. As we enter the age of space based asteroseismology for the faint subdwarf stars, we can expect our understanding of the incidence of pulsations and their amplitude variability over time and across the instability region to improve, as MOST (Walker et al. 2003) and CoRoT (Michel et al. 2008) have done for brighter pulsating stars. Meanwhile, a new generation of ground based instruments are coming on-line, capable of high temporal resolution and simultaneous multi-colour photometry, by splitting the light with dichroics and reading out several CCDs simultaneously. ULTRACAM has pioneered this technology, permitting reliable amplitude ratios to be measured and used directly for mode identification.

As theoretical models of the interior structure of these stars become sophisticated enough to accurately predict the pulsation frequencies ( $\mathrm{Hu}$ et al. 2008, 2009), new high-precision photometric observations will help us distinguish between the possible formation scenarios. When this happens we will finally be in position to unravel the complete evolutionary history of the hot subdwarf stars.

Acknowledgements. The authors thank the staff at the NOT for excellent support over a decade of observations.

The time-series data presented here have been taken using ALFOsC, which is owned by the Instituto de Astrofisica de Andalucia (IAA) and operated at the Nordic Optical Telescope under agreement between IAA and the NBIfAFG of the Astronomical Observatory of Copenhagen.

The research leading to these results has received funding from the European Research Council under the European Community's Seventh Framework Programme (FP7/2007-2013)/ERC grant agreement № 227224 (PROSPERITY), as well as from the Research Council of K.U. Leuven grant agreement GOA/2008/04.

C.R.L. acknowledges an Ángeles Alvariño contract of the regional government Xunta de Galicia.

\section{References}

Aerts, C., Jeffery, C. S., Fontaine, G., et al. 2006, MNRAS, 367, 1317 Aguilar-Sánchez, Y. 1998, Ph.D. Thesis, University of Bonn

Allard, F., Wesemael, F., Fontaine, G., Bergeron, P., \& Lamontagne, R. 1994, AJ, 107, 1565

Baran, A., \& Fox Machado, L. 2010, Ap\&SS, accepted [arXiv: 0912 .4332]

Barlow, B. N., Dunlap, B. H., Lynas-Gray, A. E., \& Clemens, J. C. 2009, AJ, 138,686

Barlow, B. N., Dunlap, B. H., Clemens, J. C., et al. 2010, MNRAS, 403, 324

Beers, T. C., Doinidis, S. P., Griffin, K. E., Preston, G. W., \& Shectman, S. A. 1992, AJ, 103, 267

Bessell, M. S. 1990, PASP, 102, 1181

Billeres, M., Fontaine, G., Brassard, P., et al. 1997, ApJ, 487, L81

Billeres, M., Fontaine, G., Brassard, P., et al. 1998, ApJ, 494, L75

Billéres, M., Fontaine, G., Brassard, P., et al. 2000, ApJ, 530, 441

Billères, M., Fontaine, G., Brassard, P., \& Liebert, J. 2002, ApJ, 578, 515

Bixler, J. V., Bowyer, S., \& Laget, M. 1991, A\&A, 250, 370

Bonanno, A., Catalano, S., Frasca, A., Mignemi, G., \& Paternò, L. 2003, A\&A, 398,283

Brassard, P., Fontaine, G., Billères, M., et al. 2001, ApJ, 563, 1013

Charpinet, S., Fontaine, G., Brassard, P., et al. 1997, ApJ, 483, L123 
Charpinet, S., Fontaine, G., Brassard, P., et al. 2005, in PASPC, 14th European Workshop on White Dwarfs, ed. D. Koester, \& S. Moehler, 334, 619 Charpinet, S., Silvotti, R., Bonanno, A., et al. 2006, A\&A, 459, 565

Christensen-Dalsgaard, J., Arentoft, T., Brown, T. M., et al. 2007, Commun. Asteroseismol., 150, 350

Dorman, B., Rood, R. T., \& O’Connell, R. W. 1993, ApJ, 419, 596

Drechsel, H., Heber, U., Napiwotzki, R., et al. 2001, A\&A, 379, 893

Dreizler, S., Schuh, S. L., Deetjen, J. L., Edelmann, H., \& Heber, U. 2002, A\&A, 386, 249

Edelmann, H. 2003, Ph.D. Thesis, University of Erlangen-Nurnberg

Edelmann, H., Heber, U., Hagen, H.-J., et al. 2003, A\&A, 400, 939

Eisenstein, D. J., Liebert, J., Harris, H. C., et al. 2006, ApJS, 167, 40

Fontaine, G., Brassard, P., Charpinet, S., et al. 2003, ApJ, 597, 518

Fontaine, G., Brassard, P., Charpinet, S., \& Chayer, P. 2006, Mem. Soc. Astron. Ital., 77, 49

Geier, S., Nesslinger, S., Heber, U., et al. 2007, A\&A, 464, 299

Gianninas, A., Bergeron, P., \& Fontaine, G. 2007, in 15th European Workshop on White Dwarfs, ed. R. Napiwotzki, \& M. R. Burleigh, PASPC, 372, 577

Green, E. M., Fontaine, G., Reed, M. D., et al. 2003, ApJ, 583, L31

Green, E. M., Fontaine, G., Hyde, E. A., For, B.-Q., \& Chayer, P. 2008, in Hot Subdwarf Stars and Related Objects, ed. U. Heber, C. S. Jeffery, \& R. Napiwotzki, PASPC, 392, 75

Green, R. F., Schmidt, M., \& Liebert, J. 1986, ApJS, 61, 305

Hagen, H.-J., Groote, D., Engels, D., \& Reimers, D. 1995, A\&AS, 111, 195

Han, Z., Podsiadlowski, P., Maxted, P. F. L., Marsh, T. R., \& Ivanova, N. 2002, MNRAS, 336, 449

Han, Z., Podsiadlowski, P., Maxted, P. F. L., \& Marsh, T. R. 2003, MNRAS, 341, 669

Heber, U. 1986, A\&A, 155, 33

Heber, U. 2009, ARA\&A, 47, 211

Heber, U., Edelmann, H., Lemke, M., Napiwotzki, R., \& Engels, D. 1999, in 11th European Workshop on White Dwarfs, ed. J.-E. Solheim, \& E. G. Meistas, PASPC, 169, 551

Heber, U., Reid, I. N., \& Werner, K. 2000, A\&A, 363, 198

Heber, U., Moehler, S., Napiwotzki, R., Thejll, P., \& Green, E. M. 2002, A\&A, 383,938

Heber, U., Drechsel, H., Østensen, R., et al. 2004, A\&A, 420, 251

Hu, H., Dupret, M., Aerts, C., et al. 2008, A\&A, 490, 243

Hu, H., Nelemans, G., Aerts, C., \& Dupret, M.-A. 2009, A\&A, 508, 869

Jeffery, C. S., \& Saio, H. 2006, MNRAS, 372, L48

Jeffery, C. S., \& Saio, H. 2007, MNRAS, 378, 379

Kawaler, S. D., \& Hostler, S. R. 2005, ApJ, 621, 432

Kilkenny, D. 2010, Ap\&SS, submitted

Kilkenny, D., Heber, U., \& Drilling, J. S. 1988, South African Astronomical Observatory Circular, 12, 1

Kilkenny, D., Koen, C., O’Donoghue, D., \& Stobie, R. S. 1997, MNRAS, 285, 640

Kilkenny, D., O’Donoghue, D., Koen, C., Lynas-Gray, A. E., \& van Wyk, F. 1998, MNRAS, 296, 329

Kilkenny, D., Koen, C., O’Donoghue, D., et al. 1999, MNRAS, 303, 525

Kilkenny, D., Billères, M., Stobie, R. S., et al. 2002, MNRAS, 331, 399

Kilkenny, D., Reed, M. D., O'Donoghue, D., et al. 2003, MNRAS, 345, 834

Kilkenny, D., Stobie, R. S., O’Donoghue, D., et al. 2006, MNRAS, 367, 1603

Kilkenny, D., O'Donoghue, D., Crause, L., et al. 2009, MNRAS, 396, 548

Koen, C. 1998, MNRAS, 300, 567

Koen, C. 2007, MNRAS, 377, 1275

Koen, C., Kilkenny, D., O’Donoghue, D., van Wyk, F., \& Stobie, R. S. 1997, MNRAS, 285, 645

Koen, C., O’Donoghue, D., Kilkenny, D., et al. 1998a, MNRAS, 296, 317

Koen, C., O'Donoghue, D., Pollacco, D. L., \& Nitta, A. 1998b, MNRAS, 300, 1105

Koen, C., O’Donoghue, D., Kilkenny, D., Stobie, R. S., \& Saffer, R. A. 1999a, MNRAS, 306, 213

Koen, C., O’Donoghue, D., Pollacco, D. L., \& Charpinet, S. 1999b, MNRAS, 305,28

Koen, C., O’Donoghue, D., Kilkenny, D., \& Pollacco, D. L. 2004, New A, 9, 565

Kuschnig, R., Weiss, W. W., Gruber, R., Bely, P. Y., \& Jenkner, H. 1997, A\&A, 328,544

Lamontagne, R., Demers, S., Wesemael, F., Fontaine, G., \& Irwin, M. J. 2000, AJ, 119, 241

Lisker, T., Heber, U., Napiwotzki, R., et al. 2005, A\&A, 430, 223

Lutz, R., Schuh, S., Silvotti, R., Kruspe, R., \& Dreizler, S. 2009, Commun. Asteroseismol., 159, 94
Martin, D. C., Fanson, J., Schiminovich, D., et al. 2005, ApJ, 619, L1

Maxted, P. f. L., Heber, U., Marsh, T. R., \& North, R. C. 2001, MNRAS, 326, 1391

McCook, G. P., \& Sion, E. M. 1987, ApJS, 65, 603

Michel, E., Baglin, A., Weiss, W. W., et al. 2008, Commun. Asteroseismol., 156, 73

Moehler, S., de Boer, K. S., \& Heber, U. 1990, A\&A, 239, 265

Munari, U., Sordo, R., Castelli, F., \& Zwitter, T. 2005, A\&A, 442, 1127

Napiwotzki, R. 1997, A\&A, 322, 256

Napiwotzki, R., Edelmann, H., Heber, U., et al. 2001, A\&A, 378, L17

Napiwotzki, R., Karl, C. A., Lisker, T., et al. 2004, Ap\&SS, 291, 321

Nather, R. E., Winget, D. E., Clemens, J. C., Hansen, C. J., \& Hine, B. P. 1990 , ApJ, 361, 309

Noguchi, T., Maehara, H., \& Kondo, M. 1980, Annals of the Tokyo Astronomical Observatory, 18,55

O’Donoghue, D., Lynas-Gray, A. E., Kilkenny, D., Stobie, R. S., \& Koen, C. 1997, MNRAS, 285, 657

O’Donoghue, D., Koen, C., Lynas-Gray, A. E., Kilkenny, D., \& van Wyk, F. 1998, MNRAS, 296, 306

Oreiro, R., Ulla, A., Pérez Hernández, F., et al. 2004, A\&A, 418, 243

Oreiro, R., Pérez Hernández, F., Ulla, A., et al. 2005, A\&A, 438, 257

Oreiro, R., Pérez Hernández, F., Østensen, R., et al. 2007, A\&A, 461, 585

Oreiro, R., Østensen, R. H., Green, E. M., \& Geier, S. 2009, A\&A, 496, 827

Østensen, R. 2009, Commun. Asteroseismol., 159, 75

Østensen, R., \& Solheim, J.-E. 2000, Baltic Astron., 9, 411

Østensen, R., Heber, U., Silvotti, R., et al. 2001a, A\&A, 378, 466

Østensen, R., Solheim, J.-E., Heber, U., et al. 2001b, A\&A, 368, 175

Østensen, R., Oreiro, R., Drechsel, H., et al. 2007, in 15th European Workshop on White Dwarfs, ed. R. Napiwotzki, \& M. R. Burleigh, PASPC, 372, 483

Østensen, R. H. 2000, Ph.D. Thesis, University of Troms $\emptyset$

Østensen, R. H. 2004, Ap\&SS, 291, 263

Østensen, R. H. 2006, Baltic Astron., 15, 85

O’Toole, S. J., Heber, U., \& Benjamin, R. A. 2004, A\&A, 422, 1053

Piccioni, A., Bartolini, C., Bernabei, S., et al. 2000, A\&A, 354, L13

Ramsay, G., Napiwotzki, R., Hakala, P., \& Lehto, H. 2006, MNRAS, 371, 957

Randall, S. K., Green, E. M., van Grootel, V., et al. 2007, A\&A, 476, 1317

Randall, S. K., Calamida, A., \& Bono, G. 2009a, A\&A, 494, 1053

Randall, S. K., Van Grootel, V., Fontaine, G., Charpinet, S., \& Brassard, P. 2009b, A\&A, 911

Reed, M. D., \& Stiening, R. 2004, PASP, 116, 506

Reed, M. D., Eggen, J. R., Zhou, A.-Y., et al. 2006, MNRAS, 369, 1529

Reed, M. D., O’Toole, S. J., Terndrup, D. M., et al. 2007a, ApJ, 664, 518

Reed, M. D., Terndrup, D. M., Zhou, A.-Y., et al. 2007b, MNRAS, 378, 1049

Reed, M., Tendrup, D., Østensen, R., et al. 2010, Ap\&SS, submitted

Saffer, R. A., Bergeron, P., Koester, D., \& Liebert, J. 1994, ApJ, 432, 351

Schlegel, D. J., Finkbeiner, D. P., \& Davis, M. 1998, ApJ, 500, 525

Schuh, S., Huber, J., Dreizler, S., et al. 2006, A\&A, 445, L31

Silvotti, R., Solheim, J.-E., Gonzalez Perez, J. M., et al. 2000, A\&A, 359, 1068

Silvotti, R., Janulis, R., Schuh, S. L., et al. 2002a, A\&A, 389, 180

Silvotti, R., Østensen, R., Heber, U., et al. 2002b, A\&A, 383, 239

Silvotti, R., Bonanno, A., Bernabei, S., et al. 2006, A\&A, 459, 557

Silvotti, R., Schuh, S., Janulis, R., et al. 2007, Nature, 449, 189

Silvotti, R., Handler, G., Schuh, S., Castanheira, B., \& Kjeldsen, H. 2009, Commun. Asteroseismol., 159, 97

Solheim, J.-E., \& Østensen, R. 2006, Baltic Astron., 15, 231

Solheim, J.-E., Østensen, R., Silvotti, R., \& Heber, U. 2004, Ap\&SS, 291, 419

Stepanian, J. A., Green, R. F., Foltz, C. B., et al. 2001, AJ, 122, 3361

Stobie, R. S., Kawaler, S. D., Kilkenny, D., O’Donoghue, D., \& Koen, C. 1997a, MNRAS, 285, 651

Stobie, R. S., Kilkenny, D., O’Donoghue, D., et al. 1997b, MNRAS, 287, 848

Stoughton, C., Lupton, R. H., Bernardi, M., et al. 2002, AJ, 123, 485

Telting, J. H., \& Østensen, R. H. 2004, A\&A, 419, 685

Telting, J. H., Østensen, R. H., Oreiro, R., et al. 2010, Ap\&SS, submitted

Vučković, M., Kawaler, S. D., O’Toole, S., et al. 2006, ApJ, 646, 1230

Vučković, M., Aerts, C., Østensen, R., et al. 2007, A\&A, 471, 605

Walker, G., Matthews, J., Kuschnig, R., et al. 2003, PASP, 115, 1023

Winter, C. 2006, Ph.D. Thesis, Armagh Observatory \& The Queen's University of Belfast

Wisotzki, L., Koehler, T., Groote, D., \& Reimers, D. 1996, A\&AS, 115, 227

Woudt, P. A., Kilkenny, D., Zietsman, E., et al. 2006, MNRAS, 371, 1497

Young, A. T. 1993, The Observatory, 113, 41

Zacharias, N., Monet, D. G., Levine, S. E., et al. 2005, VizieR Online Data Catalog, 1297, 0

Zhou, A.-Y., Reed, M. D., Harms, S., et al. 2006, MNRAS, 367, 179 